\title{
Bilinear Entropy Expansion from the Decisional Linear Assumption
}

\author{
Lucas Kowalczyk \\ Columbia University \\ luke@cs. columbia.edu
}

\author{
Allison Bishop Lewko* \\ Columbia University \\ alewko@cs.columbia.edu
}

\begin{abstract}
We develop a technique inspired by pseudorandom functions that allows us to increase the entropy available for proving the security of dual system encryption schemes under the Decisional Linear Assumption. We show an application of the tool to Attribute-Based Encryption by presenting a Key-Policy ABE scheme that is fully-secure under DLIN with short public parameters.
\end{abstract}

\section{Introduction}

Since its conception in [32], attribute-based encryption (ABE) has served as a demonstrably fertile ground for exploring the possible tradeoffs between expressibility, security, and efficiency in cryptographically enforced access control. In addition to the potential applications it has in its own right, the primitive of attribute-based encryption has been a catalyst for the definitions and constructions of further cryptographic primitives, such as functional encryption for general circuits. The rich structure of secret keys demanded by expressive attribute-based encryption has promoted a continuing evolution of proof techniques designed to meet the challenges inherent in balancing large and complex structures on the pinhead of simple computational hardness assumptions.

The origins of attribute-based encryption can be traced back to identity-based encryption $[11,5]$, where users have identities that serve as public keys and secret keys are generated on demand by a master authority. A desirable notion of security for such schemes ensures resilience against arbitrary collusions among users by allowing an attacker to demand many secret keys for individual users and attack a ciphertext encrypted to any user not represented in the set of obtained keys. Proving this kind of security requires a reduction design that can satisfy the attacker's demands without fully knowing the master secret key. This challenge is exacerbated in the (key-policy) attribute-based setting, where user keys correspond to access policies expressed over attributes and ciphertexts are associated with subsets of these attributes. Decryption is allowed precisely when a single user's policy is satisfied by a ciphertext's attribute set. Thus, the structure of allowable keys that the attacker can request grows more complex as the scheme is equipped to express more complex policies.

As a consequence of this, the intuitive and elegant constructions of attribute-based encryption in bilinear groups in $[18,34]$ were only proven secure in the selective security model: a weakened model of security that requires the attacker to declare the target of attack in advance, before seeing the public parameters of the system. This limitation of the model allows

\footnotetext{
*Allison Lewko is supported in part by NSF CNS 1413971 and NSF CCF 1423306.
} 
the security reduction to embed the computational challenge into its view of the public parameters of the scheme in a way that partitions the space of secret keys. Keys that do not satisfy the targeted ciphertext are able to be generated under the embedding, while keys that do satisfy the ciphertext cannot be generated. This approach does not extend well to the full security model, where this artificial limitation on the attacker is lifted.

The first fully secure ABE schemes appeared in [19], using the dual system encryption methodology [33] for designing the security reduction. In a dual system approach, there are typically multiple (computationally indistinguishable) forms of keys and ciphertexts. There are "normal" keys and ciphertexts that are employed in the real system, and then are various forms of "semi-functional" keys and ciphertexts. The core idea is to prove security via a hybrid argument, where the ciphertext is changed to semi-functional and keys are changed to semi-functional types one by one, until all the keys are of a semi-functional type incapable of decrypting the semi-functional ciphertext (it is important that they still decrypt normal ciphertexts, otherwise the hybrid transitions could be detected by the attacker who can create normal ciphertexts for itself using the public parameters). Once we reach a state where the key and ciphertexts distributions provided to the attacker are no longer bound by correct decrypt behavior, it is easier for the reduction to produce these without knowing the master secret key.

The most critical step of these dual system arguments occurs when a particular key changes from a type that can decrypt the challenge ciphertext to a type that cannot - the fact that this change is not detected by the attacker is where the reduction must use the criterion that the access policy is not satisfied. The security reductions in [19] and many subsequent works (e.g. $[28,22])$ used an information-theoretic argument for this step. However, this argument requires a great deal of entropy (specifically, fresh randomness for each attribute-use in a policy). This entropy was supplied by parameters in the semi-functional space that paralleled the published parameters of the normal space. This necessitated a blowup in public parameter and ciphertext sizes, specifically a multiplicative factor of the the number of attribute-uses allowed for access policies.

In [26], it was observed that the initial steps of a typical dual system encryption hybrid argument could be re-interpreted as providing a "shadow copy" of the system parameters in the semi-functional space that does not have to be committed to when the public parameters for the normal space are provided. This perspective suggests that one can embed a computational challenge into these semi-functional space parameters as semi-functional objects are produced. For instance, when a portion of these parameters affect a single semi-functional key that is queried after the semi-functional ciphertext, one can essentially embed the challenge in the same way as the original selective security arguments in [18]. In the reverse case, where the semi-functional key is queried before the challenge ciphertext, the embedding can be similar to a selective security proof for a ciphertext-policy ABE scheme, where keys are associated with attributes and ciphertexts are associated with access policies. In [26], state of the art selective techniques for KP-ABE and CP-ABE systems were combined into a full security proof, avoiding the blowup in parameters incurred by the information-theoretic dual system techniques.

However, even selective security for CP-ABE systems remains a rather challenging task, and the state of the art technique in [34] introduces an undesirable $q$-type assumption into the fully secure ABE scheme. In the CP-ABE setting, selectivity means that the attacker declares a target access policy up front. This can then be leveraged by the security reduction to design public parameters so that it can create keys precisely for sets of attributes that do not satisfy this target policy. The $q$-type assumption in [34] was a consequence of the need to encode a potentially large access policy into small public parameters. This leaves us still searching for an ideal KP-ABE scheme in the bilinear setting that has parameter sizes comparable to the selectively secure scheme in [18] and a full security proof from a simple assumption such as the 
decisional linear assumption (DLIN). A security reduction for such a scheme must seemingly break outside the mold of using either a purely information-theoretic or purely computational argument for leveraging the fact that a requested key policy cannot be satisfied by the challenge ciphertext.

Our Results To demonstrate our approach, we present a KP-ABE constructions in the composite-order bilinear setting which is proven fully secure from simple assumptions, and supports LSSS/MSP access policies (like its bilinear predecessors). Security is proven using a few specific instances of subgroup-decision assumptions and DLIN. Our scheme greatly reduces the size of the public parameters as compared to [19, 28], as the number of group elements we need to include in the public parameters grows only logarithmically rather than linearly in the bound on the number of attribute-uses in an access policy.

Our Techniques We intermix the computational and information-theoretic dual system encryption approaches, using computational steps to "boost" the entropy of a small set of (unpublished) semi-functional parameters to a level that suffices to make the prior informationtheoretic argument work. Essentially, we use the fact that the semi-functional space parameters are never published to not only "delay" their definition as exploited in [26], but further to argue that they can (computationally) appear to provide more entropy than their size would information-theoretically allow. The gadget that allows us do this computational pre-processing before the running information-theoretic argument is presented as our "bilinear entropy expansion lemma."

The inspiration for the gadget construction comes from pseudorandom generators/pseudorandom functions. Naturally, if we want a small set of semi-functional generators to seemingly produce a large amount of entropy, we may want to view these parameters as the seed for a PRF, for example. Out-of-the-box PRF constructions like Naor-Reingold [27] and its DLIN-based extension [25] however are unsuitable in the bilinear setting (even though the DLIN version would remain secure) because they would require direct access to the seed for computation, and a secure bilinear construction will only provide indirect access to the seed as exponents of group elements.

To circumvent this difficulty, we use a subset-sum based construction that can be computed in a bilinear group with the seed elements in the exponents. Of course, using a naked linear structure would be detectable, but we are able to use a rather minimal amount of additional random exponents to push the linear sub-structure out of reach of detection by regular group or pairing operations.

We build our construction in two steps. First, we present a construction for a one-use $\mathrm{KP}-\mathrm{ABE}$ system which only supports access policies where each attribute is used at most once. This scheme achieves ciphertext and key sizes which rival those of selectively secure schemes (up to constants), while significantly reducing public parameter size. Then, we apply a standard transformation to get from a one-use system to a system which allows multiple uses of attributes in policies (the number of uses allowed per attribute is constant and fixed at setup). The overhead of this transformation is drastically mitigated by our scheme's small public parameters. The effect on ciphertext and key sizes compared to previous applications of this transformation remain the same up to constants.

Further Discussion of Related Work Additional work on ABE in the bilinear setting includes various constructions of $\mathrm{KP}-\mathrm{ABE}$ and $\mathrm{CP}-\mathrm{ABE}$ schemes (e.g. [4, 31, 17]), schemes supporting multiple authorities (e.g. [7, 8, 30, 22]), and schemes supporting large attribute universes (e.g. [23, 29]). Some of the structure for randomization in our schemes is inspired by 
[23]. The large universe scheme in [29] also achieves full-security with short public parameters using conceptually different techniques. We view the main contribution of this paper to be the entropy expansion lemma, which we believe is modular and potentially useful in other settings. Our approach lends a clear understanding of the roles of information-theoretic and computational techniques in dual-system encryption proofs.

There are also recent constructions of ABE schemes in the lattice setting. The construction of [16] allows access policies to be expressed as circuits, which makes it more expressive than any known bilinear scheme. It was proven selectively secure under the standard LWE assumption. Circuit policies are also supported by the construction in [13] based on multilinear maps. This scheme is also proven selectively secure, under a particular computational hardness assumption for multilinear groups. The very recent multilinear scheme in [14] achieves full security, relying on computational hardness assumptions in multilinear groups. The fully secure general functional encryption scheme in [35], which relies on indistinguishability obfuscation, can also be specialized to the ABE setting.

Some relationships between $\mathrm{ABE}$ and other cryptographic primitives have also been explored. The work of [2] derives schemes for verifiable computation from attribute-based encryption schemes, while [15] use attribute-based encryption as a tool in designing more general functional encryption and reusable garbling schemes. Dual system encryption proof techniques have also been further studied in the works of $[21,10,35,1]$, applied to achieve leakage resilience in $[24,20,12]$, and applied directly to computational assumptions in [9].

\section{Preliminaries}

\subsection{Composite Order Bilinear Groups}

Our construction uses composite order bilinear groups., which were introduced in [6]. We let $\mathcal{G}$ denote a group generator - an algorithm which takes a security parameter $\lambda$ as input and outputs a description of a bilinear group $G$. We define $\mathcal{G}$ 's output as $\left(N, G, G_{T}, e\right)$, where $N=p q w$ is a product of three distinct primes, $G$ and $G_{T}$ are cyclic groups of order $N$, and $e: G \times G \rightarrow G_{T}$ is a map with the following properties:

1. (Bilinear) $\forall g, f \in G, a, b \in \mathbb{Z}_{N}, e\left(g^{a}, f^{b}\right)=e(g, f)^{a b}$

2. (Non-degenerate) $\exists g \in G$ such that $e(g, g)$ has order $N$ in $G_{T}$.

We refer to $G$ as the source group and $G_{T}$ as the target group. We assume that the group operations in $G$ and $G_{T}$ and the map $e$ are computable in polynomial time with respect to $\lambda$, and the group descriptions of $G$ and $G_{T}$ include a generator of each group. We let $G_{p}, G_{q}$, and $G_{w}$ denote the subgroups of order $p, q$, and $w$ in $G$ respectively. We note that these subgroups are "orthogonal" to each other under the bilinear map $e$ : if $u, v$ are elements of two different subgroups out of $\left\{G_{p}, G_{q}, G_{w}\right\}$, then $e(u, v)$ is the identity element in $G_{T}$. If $g_{p}$ generates $G_{p}, g_{q}$ generates $G_{q}$, and $g_{w}$ generates $G_{w}$, then every element $f$ of $G$ can be expressed as $f=g_{p}^{c_{1}} g_{q}^{c_{2}} g_{w}^{c_{3}}$ for some values $c_{1}, c_{2}, c_{3} \in \mathbb{Z}_{N}$. We will refer to $g_{p}^{c_{1}}$ as the " $G_{p}$ part of $f$ ", for example.

\subsection{Complexity Assumptions}

We now present the complexity assumptions we will use to prove the security of our system. We use the notation $x \leftarrow S$ to express that element $x$ is chosen uniformly at random from the finite set $S$. We will first consider groups $G$ whose orders are products of three distinct primes: $p, q, w$. We denote the subgroups of $G$ with these orders by $G_{p}, G_{q}, G_{w}$ respectively. We use the notation $g_{p}, g_{q}, g_{w}$ to denote generators of these respective subgroups. 
Subgroup Decision Assumption 1 The Subgroup Decision Problem 1 is stated as follows: on a group $G$ of order $p q w$, given $g_{p}, g_{w}$ and $T$ where either $T=g_{p}^{s} \leftarrow G_{p}$ or $T=g_{p}^{s} g_{q}^{\tilde{s}} \leftarrow G_{p q}$ (where $s$ is distributed uniformly in $\mathbb{Z}_{p}$ and $\tilde{s}$ is distributed uniformly in $\mathbb{Z}_{q}$ ), output "yes" if $T=g_{p}^{s}$, and "no" otherwise.

Definition 1. Subgroup Decision Assumption 1 in $G$ : no polynomial time algorithm can achieve non-negligible advantage in deciding the Subgroup Decision Problem 1 in G.

Subgroup Decision Assumption 2 The Subgroup Decision Problem 2 is stated as follows: on a group $G$ of order $p q w$, given $g_{p}, g_{w}, g_{p}^{s} g_{q}^{\tilde{s}}, g_{q}^{\tilde{r}} g_{w}^{r^{*}}$ and $T$ where either $T=g_{p}^{y} g_{w}^{y^{*}}$ or $T=g_{p}^{y} g_{q}^{\tilde{y}} g_{w}^{y^{*}}$ (where $s, y$ are distributed uniformly in $\mathbb{Z}_{p}, \tilde{s}, \tilde{r}$, and $\tilde{y}$ are distributed uniformly in $\mathbb{Z}_{q}$, and $r^{*}, y^{*}$ are distributed uniformly in $\mathbb{Z}_{w}$ ), output "yes" if $T=g_{p}^{y} g_{w}^{y^{*}}$, and "no" otherwise.

Definition 2. Subgroup Decision Assumption 2 in $G$ : no polynomial time algorithm can achieve non-negligible advantage in deciding the Subgroup Decision Problem 2 in $G$.

Subgroup Decision Assumption 3 The Subgroup Decision Problem 3 is stated as follows: given a group $G$ of order $p q w$, given $g_{p}, g_{q}, g_{w}, g_{p}^{\alpha} g_{q}^{\tilde{\alpha}}, g_{p}^{s} g_{q}^{\tilde{s}}$, and $T$ where either $T=e\left(g_{p}, g_{p}\right)^{\alpha s}$ or $T=X$ (where $\alpha, s$ are distributed uniformly in $\mathbb{Z}_{p}, \tilde{\alpha}, \tilde{s}$ are distributed uniformly in $\mathbb{Z}_{q}$, and $\mathrm{X}$ is distributed uniformly in $\left.G_{T}\right)$, output "yes" if $T=e\left(g_{p}, g_{p}\right)^{\alpha s}$, and "no" otherwise.

Definition 3. Subgroup Decision Assumption 3 in $G$ : no polynomial time algorithm can achieve non-negligible advantage in deciding the Subgroup Decision Problem 3 problem in G.

We additionally use one assumption in the prime order setting, where $G$ is a bilinear group of prime order $q$ and $g$ is a generator:

2-Linear Assumption (DLIN) The 2-Linear problem is stated as follows: given a cyclic group $G$ of prime order $p, g, g^{y_{1}}, g^{y_{2}}, g^{y_{1} c_{1}}, g^{y_{2} c_{2}}, g^{c_{1}+c_{2}+r} \in G$ (where $y_{1}, y_{2}, c_{1}, c_{2}$ are distributed uniformly in $\mathbb{Z}_{p}$ and $r$ is either a uniform random element of $\mathbb{Z}_{p}$ or 0 ), output "yes" if $r$ is a random element of $\mathbb{Z}_{p}$ and "no" otherwise.

Definition 4. 2-Linear Assumption in G: no polynomial time algorithm can achieve nonnegligible advantage in deciding the 2-Linear problem in $G$.

\subsection{Background for ABE}

We now give required background material on Linear Secret Sharing Schemes, the formal definition of a KP-ABE scheme, and the security definition we will use.

\subsubsection{Linear Secret Sharing Schemes}

Our construction uses linear secret-sharing schemes (LSSS). We use the following definition (adapted from [3]). In the context of ABE, attributes will play the role of parties and will be represented as nonempty subsets $K \subseteq[k]$ for a fixed $k$.

Definition 5. (Linear Secret-Sharing Schemes (LSSS)) A secret sharing scheme $\Pi$ over a set of attributes is called linear (over $\mathbb{Z}_{p}$ ) if

1. The shares belonging to all attributes form a vector over $\mathbb{Z}_{p}$. 
2. There exists an $\ell \times n$ matrix $\Lambda$ called the share-generating matrix for $\Pi$. The matrix $\Lambda$ has $\ell$ rows and $n$ columns. For all $j=1, \ldots, \ell$, the $j^{\text {th }}$ row of $\Lambda$ is labeled by an attribute $K$. When we consider the column vector $v=\left(s, r_{2}, \ldots, r_{n}\right)$, where $s \in \mathbb{Z}_{p}$ is the secret to be shared and $r_{2}, \ldots, r_{n} \in \mathbb{Z}_{p}$ are randomly chosen, then $\Lambda v$ is the vector of $\ell$ shares of the secret $s$ according to $\Pi$. The share $(\Lambda v)_{j}=\lambda_{K}$ belongs to attribute $K$.

We note the linear reconstruction property: we suppose that $\Pi$ is an LSSS. We let $S$ denote an authorized set. Then there is a subset $S^{*} \subseteq S$ such that the vector $(1,0, \ldots, 0)$ is in the span of rows of $\Lambda$ indexed by $S^{*}$, and there exist constants $\left\{\omega_{K} \in \mathbb{Z}_{p}\right\}_{K \in S^{*}}$ such that, for any valid shares $\left\{\lambda_{K}\right\}$ of a secret $s$ according to $\Pi$, we have: $\sum_{K \in S^{*}} \omega_{K} \lambda_{K}=s$. These constants $\left\{\omega_{K}\right\}$ can be found in time polynomial in the size of the share-generating matrix $\Lambda$ [3]. For unauthorized sets, no such $S^{*},\left\{\omega_{K}\right\}$ exist.

For our composite order group construction, we will employ LSSS matrices over $\mathbb{Z}_{N}$, where $N$ is a product of three distinct primes $p, q, w$. As in the definition above over the prime order $\mathbb{Z}_{p}$, we say a set of attributes $S$ is authorized if is a subset $S^{*} \subseteq S$ such that the rows of the access matrix $A$ labeled by elements of $S$ have the vector $(1,0, \ldots, 0)$ in their span modulo $N$. However, in our security proof for our composite order system, we will further assume that for an unauthorized set, the corresponding rows of $A$ do not include the vector $(1,0, \ldots, 0)$ in their span modulo $q$. We may assume this because if an adversary can produce an access matrix $A$ over $\mathbb{Z}_{N}$ and an unauthorized set over $\mathbb{Z}_{N}$ that is authorized over $\mathbb{Z}_{q}$, then this can be used to produce a non-trivial factor of the group order $N$, which would violate our subgroup decision assumptions.

\subsubsection{KP-ABE Definition}

A key-policy attribute-based encryption system consists of four algorithms: Setup, Encrypt, KeyGen, and Decrypt.

$\operatorname{Setup}(\lambda, \mathcal{U}) \rightarrow(\mathrm{PP}, \mathrm{MSK})$ The setup algorithm takes in the security parameter $\lambda$ and the attribute universe description $\mathcal{U}$. It outputs the public parameters $\mathrm{PP}$ and a master secret key MSK.

Encrypt(PP, $M, S) \rightarrow \mathrm{CT}$ The encryption algorithm takes in the public parameters $\mathrm{PP}$, the message $M$, and a set of attributes $S$. It will output a ciphertext CT. We assume that $S$ is implicitly included in CT.

KeyGen(MSK, PP, $\mathbb{A}) \rightarrow$ SK The key generation algorithm takes in the master secret key MSK, the public parameters PP, and an access structure $\mathbb{A}$ over the universe of attributes. It outputs a private key SK which can be used to decrypt ciphertexts encrypted under a set of attributes which satisfies $\mathbb{A}$. We assume that $\mathbb{A}$ is implicitly included in SK.

$\operatorname{Decrypt}(\mathrm{PP}, \mathrm{CT}, \mathrm{SK}) \rightarrow M$ The decryption algorithm takes in the public parameters PP, a ciphertext CT encrypted under a set of attributes $S$, and a private key SK for an access structure $\mathbb{A}$. If the set of attributes of the ciphertext satisfies the access structure of the private key, it outputs the message $M$.

\subsubsection{Full Security for KP-ABE Systems}

We define full security for KP-ABE Systems in terms of the following game: 
Setup The challenger runs the Setup algorithm and gives the public parameters to the attacker.

Phase 1 The attacker queries the challenger for private keys corresponding to access structures.

Challenge The attacker declares two equal length messages $M_{0}, M_{1}$ and a set of attributes $A \subseteq \mathcal{U}$ where $\mathcal{U}$ is the attribute universe such that $A$ does not satisfy the access structure of any of the keys requested in Phase 1 . The challenger flips a random coin $\beta \in\{0,1\}$, encrypts $M_{\beta}$ under $S$ to yield ciphertext $\mathrm{CT}_{\beta}$ and gives $\mathrm{CT}_{\beta}$ to the attacker.

Phase 2 The attacker queries the challenger for private keys corresponding to access structures that are not satisfied by $S$.

Guess The attacker outputs a guess $\beta^{\prime}$.

Definition 6. The advantage of an attacker $\mathcal{A}$ in this game is defined as $A d v_{\mathcal{A}}^{K P-A B E}(\lambda)=$ $\operatorname{Pr}\left[\beta=\beta^{\prime}\right]-\frac{1}{2}$.

Definition 7. A key-policy attribute based encryption scheme is fully secure if no polynomial time algorithm can achieve a non-negligible advantage in the above security game.

\subsubsection{Transformation from One-Use to Multiple Use KP-ABE}

Given a KP-ABE scheme which is fully-secure when attributes are used at most once in access policies, we can obtain a KP-ABE scheme which is fully-secure when each attribute is used at most some constant number of times in access policies using a standard transformation. Essentially, multiple uses of an attribute are treated as new "attributes" in the one-use system. For example, if we want an attribute $x$ to be able to be used up to $k_{x}$ times in access policies, we will instantiate our one-use system with $k_{x}$ "attributes" $x: 1, \ldots, x: k_{x}$. Each time we want to label a row of an access matrix $\Lambda$ with $x$, we label it with $x: i$ for a new value of $i$. Each time we want to associate a subset $S$ of attributes to a ciphertext, we instead use the set $S^{\prime}=\left\{x: 1, \ldots, x: k_{x} \mid x \in S\right\}$. We can then employ the one-use KP-ABE scheme on this new larger set of "attributes" and retain its full security and functionality.

Clearly, this transformation comes at a cost. Typically, the ciphertext and public parameter size of the KP-ABE scheme resulting from the transformation now scale linearly with the number of attribute-uses allowed in access policies, not just the number of attributes. This blowup is seen in all previous fully secure KP-ABE schemes based on static assumptions and presents a problem if one desires policies which have high reuse of attributes. Our one-use KP-ABE scheme mitigates the problem with public parameter size by featuring public parameters that scale only logarithmically with the number of attributes supported by the system, compared to the linear scaling of all other known fully secure KP-ABE schemes based on static assumptions.

\section{KP-ABE Construction}

Our single-use KP-ABE construction assumes a polynomially sized attribute universe $\mathcal{U}$ where attributes are non-empty subsets $K \subseteq[k]$ for some fixed $k$. The prior fully secure single-use $\mathrm{KP}-\mathrm{ABE}$ scheme in [19] required a fresh group element to appear in the public parameters for each attribute in the universe. After using the generic transformation discussed in section 2.3.4, 
this results in the scheme requiring a fresh group element for each attribute-use allowed in access policies. As a concrete example, if one wanted to allow 9 attributes to be used up to 7 times each, one needed to have $9 \times 7=63$ group elements in the public parameters corresponding to this attribute. In our composite order scheme, to allow the same $63=2^{6}-1$ attribute-uses, we only need $2 \times 6$ group elements in the public parameters corresponding to the attribute. The way we accomplish this dramatic "compression" of public parameters is to note that the encryptor can produce 63 group elements from 6 by taking products of all non-empty subsets (these correspond to subset-sums in the exponent). More generally, given $k$ group elements $g^{a_{1}}, \ldots, g^{a_{k}}$, we can produce $2^{k}-1$ group elements by enumerating over all non-empty subsets $K \subseteq[k]$ and computing $g^{j \in K} a_{j}$. We name the resulting collection of elements $g^{A_{K}}$, where $A_{K}:=\sum_{j \in K} a_{j}$. Our composite order scheme uses two parallel such subset constructions (which is the reason for the factor of 2).

Obviously, these $2^{k}-1$ group elements no longer look random - they have linear relationships in their exponents by construction. However, since we are assuming the decisional linear assumption is hard, if we choose $2^{k}-1$ additional random exponents $\left\{t_{K}\right\}$, then the $2\left(2^{k}-1\right)$ group elements formed as $\left\{g^{t_{K}}, g^{t_{K} A_{K}}\right\}$ are computationally indistinguishable from $2\left(2^{k}-1\right)$ uniformly random group elements (which lack any hidden linear structures in their exponents). The proof of this is the core of bilinear entropy expansion lemma, though the full statement of the lemma includes some additional structure that is useful for linking into a KP-ABE construction. The dual system encryption framework allows us to apply this argument to the parameters in the semi-functional space, where we do not need to publish the values $\left\{g^{a_{j}}\right\}$. (Note that publishing these would make the structure of $\left\{g^{t_{K}}, g^{t_{K} A_{K}}\right\}$ detectable through applications of the bilinear map.)

$\operatorname{Setup}(\lambda, \mathcal{U}, k) \rightarrow P P, M S K$ The setup algorithm chooses a bilinear group $G$ of order $N=$ $p q w$ where $p, q, w$ are primes. We let $G_{p}, G_{q}, G_{w}$ represent the subgroup of order $p, q$, and $w$ respectively in $G$. It then draws $\alpha \leftarrow \mathbb{Z}_{N}$ and random group element (generator) $g_{p} \in G_{p}$. For each $j \in[k]$, it chooses values $a_{j}, b_{j} \leftarrow \mathbb{Z}_{N}$.

The public parameters are $N, g_{p}, e\left(g_{p}, g_{p}\right)^{\alpha},\left\{g_{p}^{a_{j}}, g_{p}^{b_{j}}: j \in[k]\right\}$. The MSK is $\alpha$ and a generator $g_{w}$ of $G_{w}$. Such a construction is equipped to create keys for access policies which include attributes $K \subseteq[k]$ where $K$ is not empty.

KeyGen $(M S K, \Lambda, P P) \rightarrow S K$ The key generation algorithm takes in the public parameters, master secret key, and LSSS access matrix $\Lambda$. First, the key generation algorithm generates $\left\{\lambda_{K}\right\}$ : a linear sharing of $\alpha$ according to policy matrix $\Lambda$ (the reader is referred to section 2.3.1 for details). For each attribute $K$ corresponding to a row in the policy matrix $\Lambda$, it then raises generator $g_{w}$ to random exponents to create $g_{w}^{z_{K}}, g_{w}^{z_{K}^{\prime}}, g_{w}^{z_{K}^{\prime \prime}} \in G_{w}$, chooses exponent $y_{K} \leftarrow \mathbb{Z}_{N}$ and computes $g_{p}^{A_{K}}=\prod_{j \in K} g_{p}^{a_{j}}$ and $g_{p}^{B_{K}}=\prod_{j \in K} g_{p}^{b_{j}}$. Note that here and throughout the rest of the description of our construction and its proof of security we will use the notation $A_{K}=\sum_{j \in K} a_{j}$ and $B_{K}=\sum_{j \in K} b_{j}$. It then outputs the secret key:

$$
S K_{\Lambda}=\left\{g_{p}^{\lambda_{K}} g_{p}^{y_{K} A_{K}} g_{w}^{z_{K}}, \quad g_{p}^{y_{K}} g_{w}^{z_{K}^{\prime}}, \quad g_{p}^{y_{K} B_{K}} g_{w}^{z_{K}^{\prime \prime}}:(\forall K \text { labels } \in \Lambda)\right\}
$$

(This implicitly includes $\Lambda$ ) 
$\operatorname{Encrypt}(M, S, P P) \rightarrow C T$ The encryption algorithm first draws $s \leftarrow \mathbb{Z}_{N}$. For each $K \in S$, the encryption algorithm draws $t_{K} \leftarrow \mathbb{Z}_{N}$ and computes $g_{p}^{A_{K}}=\prod_{j \in K} g_{p}^{a_{j}}$ and $g_{p}^{B_{K}}=\prod_{j \in K} g_{p}^{b_{j}}$. It then outputs the ciphertext:

$$
C T=M e\left(g_{p}, g_{p}\right)^{\alpha s}, \quad\left\{g_{p}^{s}, \quad g_{p}^{s A_{K}} g_{p}^{t_{K} B_{K}}, \quad g_{p}^{t_{K}}:(\forall K \in S)\right\}
$$

(This implicitly includes $S$ )

$\operatorname{Decrypt}(C T, S K, P P) \rightarrow M$ We let $S$ correspond to the set of attributes associated to ciphertext $C T$, and $\Lambda$ be the policy matrix. If $S$ satisfies $\Lambda$, the decryption algorithm computes suitable constants $\omega_{K}$ such that $\sum_{K \in S^{*}} \omega_{K} \lambda_{K}=\alpha$ (recall section 2.3.1). It then computes:

$$
\begin{aligned}
& \prod_{K \in S^{*}}\left(e\left(g_{p}^{s}, g_{p}^{\lambda_{K}} g_{p}^{y_{K} A_{K}} g_{w}^{z_{K}}\right)\left(\frac{e\left(g_{p}^{y_{K}} g_{w}^{z_{K}^{\prime}}, g_{p}^{s A_{K}} g_{p}^{t_{K} B_{K}}\right)}{e\left(g_{p}^{t_{K}}, g_{p}^{y_{K} B_{K}} g_{w}^{z_{K}^{\prime \prime}}\right)}\right)^{-1}\right)^{\omega_{K}} \\
= & \prod_{K \in S^{*}}\left(e\left(g_{p}, g_{p}\right)^{s \lambda_{K}} e\left(g_{p}, g_{p}\right)^{s y_{K} A_{K}}\left(\frac{e\left(g_{p}, g_{p}\right)^{s y_{K} A_{K}} e\left(g_{p}, g_{p}\right)^{t_{K} y_{K} B_{K}}}{e\left(g_{p}, g_{p}\right)^{t_{K} y_{K} B_{K}}}\right)^{-1}\right)^{\omega_{K}} \\
= & \prod_{K \in S^{*}}\left(\frac{e\left(g_{p}, g_{p}\right)^{s \lambda_{K}} e\left(g_{p}, g_{p}\right)^{s y_{K} A_{K}}}{e\left(g_{p}, g_{p}\right)^{s y_{K} A_{K}}}\right)^{\omega_{K}} \\
= & \prod_{K \in S^{*}}\left(e\left(g_{p}, g_{p}\right)^{s \lambda_{K}}\right)^{\omega_{K}} \\
= & e\left(g_{p}, g_{p}\right)^{K \in S^{*}} s \omega_{K} \lambda_{K} \\
= & e\left(g_{p}, g_{p}\right)^{\alpha s}
\end{aligned}
$$

The message can then be recovered by computing: $M e\left(g_{p}, g_{p}\right)^{\alpha s} / e\left(g_{p}, g_{p}\right)^{\alpha s}=M$. This demonstrates correctness of the scheme.

\section{Security Proof}

Our security proof uses a hybrid argument over a sequence of games. We let Game real $_{\text {denote }}$ the real security game. The rest of the games use semi-functional keys and ciphertexts, which we describe below. We let $g_{q}$ denote a fixed generator of the subgroup $G_{q}$, which will serve as the "semi-functional space."

Like a typical dual system encryption proof, we will begin by transitioning from a normal ciphertext to a semi-functional ciphertext with semi-functional components that mimic the structure of their normal counterparts. This kind of transition can be done with a basic subgroup decision assumption. We will then perform a hybrid over keys, gradually changing each one to a semi-functional form that does not properly decrypt the semi-functional ciphertext. To start, we can bring in semi-functional components for a particular key that mimic the structure of normal components, up to the constraint that the shared valued in the semi-functional space will be 0 (modulo $q$ ). Technically, this constraint arises because we will be taking a challenge term from a subgroup decision assumption that has an unknown exponent in the normal space and raising it to a share - so we have to make this a share of 0 and separately share the $\alpha$ value in the normal space so that the unknown exponent does not affect the correctness of the 
sharing in the normal space. At a higher level, this constraint explains why the simulator at this stage of the hybrid cannot solve the challenge problem for itself by test decrypting against a semi-functional ciphertext. Since the structure in the semi-functional space parallels the normal structure and the shared value here is zero, the semi-functional components will cancel out upon decryption.

So we can arrive at a stage where a key and ciphertext have semi-functional components structured just like the normal space, but with fresh parameters modulo $q$ that are independent of the published parameters modulo $p$. This is a consequence of the Chinese Remainder Theorem, that ensures when we sample an exponent uniformly at random modulo $N$, its modulo $p$ and modulo $q$ reductions are independent and uniformly random in $\mathbb{Z}_{p}, \mathbb{Z}_{q}$ respectively. Since these implicit parameters in the semi-functional space are never published, we can use our bilinear entropy expansion lemma to argue that their subset-sum structure is hidden under the decisional linear assumption. This allows us to replace them with higher entropy parameters (lacking the subset-sum structure of the normal space), and then argue that the shared value in the semi-functional space is information-theoretically hidden (this is where we use that the access policy is not satisfied and that attributes are used at most once in the policy). This enables us to switch the semi-functional shares in the key to shares of a random value, now destroying correct decryption of a semi-functional ciphertext. We then remove some of the other (now unnecessary) semi-functional components of the key, to reclaim the entropy of those parameters to use in processing the next key in the hybrid. Finally, once we have reached a game where all keys are semi-functional with shares of a random secret modulo $q$, we can use Subgroup Decision Assumption 3 to create such keys without knowing the master secret and can hence complete the proof.

We now formally present our definitions of semi-functional ciphertexts and keys and our hybrid proof:

Semi-functional Ciphertext We will use 3 types of semi-functional ciphertexts. To produce a semi-functional ciphertext for an attribute set $S$, one first calls the normal encryption algorithm to produce a normal ciphertext consisting of:

$$
M e\left(g_{p}, g_{p}\right)^{\alpha s}, \quad\left\{g_{p}^{s}, \quad g_{p}^{s A_{K}} g_{p}^{t_{K} B_{K}}, \quad g_{p}^{t_{K}}:(\forall K \in S)\right\}
$$

One then draws $\tilde{s} \leftarrow \mathbb{Z}_{N}$. For each $K \in S$, an exponent $\tilde{t}_{K} \leftarrow \mathbb{Z}_{N}$ is chosen. The remaining composition of the semifunctional ciphertext depends on the type of ciphertext desired:

Type 1 The semi-functional ciphertext of Type 1 is formed as:

$$
M e\left(g_{p}, g_{p}\right)^{\alpha s}, \quad\left\{g_{p}^{s} g_{q}^{\tilde{s}}, \quad g_{p}^{s A_{K}} g_{p}^{t_{K} B_{K}} g_{q}^{\tilde{s} A_{K}} g_{q}^{\tilde{t}_{K} B_{K}}, \quad g_{p}^{t_{K}} g_{q}^{\tilde{t}_{K}}:(\forall K \in S)\right\}
$$

(again, here $A_{K}=\sum_{j \in K} a_{j}$ and $B_{K}=\sum_{j \in K} b_{j}$ )

Type 2 The semi-functional ciphertext of Type 2 is formed as:

$$
M e\left(g_{p}, g_{p}\right)^{\alpha s}, \quad\left\{g_{p}^{s} g_{q}^{\tilde{s}}, \quad g_{p}^{s A_{K}} g_{p}^{t_{K} B_{K}} g_{q}^{\tilde{s} A_{K}} g_{q}^{\tilde{t}_{K} \tilde{b}_{K}}, \quad g_{p}^{t_{K}} g_{q}^{\tilde{t}_{K}}:(\forall K \in S)\right\}
$$

for fixed $\tilde{b}_{K} \in \mathbb{Z}_{N}$ which are chosen uniformly at random and fixed if they do not already exist (in a semi-functional key, for instance). 
Type 3 The semi-functional ciphertext of Type 3 is formed as:

$$
M e\left(g_{p}, g_{p}\right)^{\alpha s}, \quad\left\{g_{p}^{s} g_{q}^{\tilde{s}}, \quad g_{p}^{s A_{K}} g_{p}^{t_{K} B_{K}} g_{q}^{\tilde{s} \tilde{a}_{K}} g_{q}^{\tilde{t}_{K} \tilde{b}_{K}}, \quad g_{p}^{t_{K}} g_{q}^{\tilde{t}_{K}}:(\forall K \in S)\right\}
$$

for fixed $\tilde{a}_{K}, \tilde{b}_{K} \in \mathbb{Z}_{N}$ which are chosen uniformly at random and fixed if they do not already exist.

Semi-functional Keys We will use 7 types of semi-functional keys. To produce a semifunctional key for an access policy $\Lambda$, one first calls the normal key generation algorithm to produce a normal key consisting of:

$$
\left\{g_{p}^{\lambda_{K}} g_{p}^{y_{K} A_{K}} g_{w}^{z_{K}}, \quad g_{p}^{y_{K}} g_{w}^{z_{K}^{\prime}}, \quad g_{p}^{y_{K} B_{K}} g_{w}^{z_{K}^{\prime \prime}}:(\forall K \text { labels } \in \Lambda)\right\}
$$

The first 6 types of keys fall under 3 classes which have two variants each: a "Z" variant and an "R" variant. For Z-type keys one computes a linear sharing of 0 under access policy $\Lambda$, creating shares $\tilde{\lambda}_{K}$. For R-type keys one computes a linear sharing of a random element $u$ of $\mathbb{Z}_{q}$ which is fixed once it is created and used for all R-type keys. $u$ is shared under access policy $\Lambda$, to create shares $\tilde{\lambda}_{K}$. The next steps depend on the class of the key:

Class 1 First compute $g_{q}^{A_{K}}$ and $g_{q}^{B_{K}}$ (where, again, $A_{K}$ and $B_{K}$ represent the subset-sums of $a_{j}$ and $b_{j}$ ). For each $K$ label in the honest key, one then draws $\tilde{y}_{K} \leftarrow \mathbb{Z}_{N}$ and forms the semi-functional key of type $1 \mathrm{Z}$ or $1 \mathrm{R}$ (depending on the sharing $\tilde{\lambda}_{K}$ ) as:

$$
\left\{g_{p}^{\lambda_{K}} g_{p}^{y_{K} A_{K}} g_{q}^{\tilde{\lambda}_{K}} g_{q}^{\tilde{y}_{K} A_{K}} g_{w}^{z_{K}}, \quad g_{p}^{y_{K}} g_{q}^{\tilde{y}_{K}} g_{w}^{z_{K}^{\prime}}, \quad g_{p}^{y_{K} B_{K}} g_{q}^{\tilde{y}_{K} B_{K}} g_{w}^{z_{K}^{\prime \prime}}:(\forall K \text { labels } \in \Lambda)\right\}
$$

Class 2 First compute $g_{q}^{A_{K}}$. Random values $\tilde{b}_{K} \in \mathbb{Z}_{N}$ are chosen if they do not already exist (in a semi-functional ciphertext, for instance) and fixed. For each $K$ label in the honest key, one then draws $\tilde{y}_{K} \leftarrow \mathbb{Z}_{N}$ and forms the semi-functional key of type $2 \mathrm{Z}$ or $2 \mathrm{R}$ as:

$$
\left\{g_{p}^{\lambda_{K}} g_{p}^{y_{K} A_{K}} g_{q}^{\tilde{\lambda}_{K}} g_{q}^{\tilde{y}_{K} A_{K}} g_{w}^{z_{K}}, \quad g_{p}^{y_{K}} g_{q}^{\tilde{y}_{K}} g_{w}^{z_{K}^{\prime}}, \quad g_{p}^{y_{K} B_{K}} g_{q}^{\tilde{y}_{K} \tilde{b}_{K}} g_{w}^{z_{K}^{\prime \prime}}:(\forall K \text { labels } \in \Lambda)\right\}
$$

Class 3 Random values $\tilde{a}_{K}, \tilde{b}_{K} \in \mathbb{Z}_{N}$ are chosen if they do not already exist and fixed. For each $K$ label in the honest key, one then draws $\tilde{y}_{K} \leftarrow \mathbb{Z}_{N}$ and forms the semi-functional key of type $3 \mathrm{Z}$ or $3 \mathrm{R}$ as:

$$
\left\{g_{p}^{\lambda_{K}} g_{p}^{y_{K} A_{K}} g_{q}^{\tilde{\lambda}_{K}} g_{q}^{\tilde{y}_{K} \tilde{a}_{K}} g_{w}^{z_{K}}, \quad g_{p}^{y_{K}} g_{q}^{\tilde{y}_{K}} g_{w}^{z_{K}^{\prime}}, \quad g_{p}^{y_{K} B_{K}} g_{q}^{\tilde{y}_{K} \tilde{b}_{K}} g_{w}^{z_{K}^{\prime \prime}}:(\forall K \text { labels } \in \Lambda)\right\}
$$

Note that we now have defined 6 types of keys: 1Z, 1R, 2Z, 2R, 3Z, and 3R, where the letter $(\mathrm{Z} / \mathrm{R})$ describes whether the $\tilde{\lambda}_{K}$ share zero or a random element of $\mathbb{Z}_{q}$ respectively, and the number $(1 / 2 / 3)$ describes whether the semi-functional analogues of the $g_{p}^{A_{K}}$ and $g_{p}^{B_{K}}$ in the $G_{q}$ group are structured as subset-sums or as random elements of $G_{q}$ (Class 1 keys have both $g_{q}^{A_{K}}$ and $g_{q}^{B_{K}}$. Class 2 keys have just $g_{q}^{A_{K}}$ structured, with a random element $g_{q}^{\tilde{b}_{K}}$. Class 3 keys have both replaced by random elements $g_{q}^{\tilde{a}_{K}}, g_{q}^{\tilde{b}_{K}}$ of $G_{q}$ ). There is one final type of key, type $4 \mathrm{R}$, which does not contain any of these elements:

Type $4 \mathbf{R}$ Using shares $\tilde{\lambda}_{K}$ of $u$ (which is randomly chosen from $\mathbb{Z}_{p}$ and fixed if it has not already been fixed), one forms the semi-functional key of type $4 \mathrm{R}$ as:

$$
\left\{g_{p}^{\lambda_{K}} g_{p}^{y_{K} A_{K}} g_{q}^{\tilde{\lambda}_{K}} g_{w}^{z_{K}}, \quad g_{p}^{y_{K}} g_{w}^{z_{K}^{\prime}}, \quad g_{p}^{y_{K} B_{K}} g_{w}^{z_{K}^{\prime \prime}}:(\forall K \text { labels } \in \Lambda)\right\}
$$


Proof Structure Our hybrid proof takes place over a series of games defined as follows: Letting $Q$ denote the total number of key queries that the attacker makes, we define Game $_{\ell_{1}}$, Game $_{\ell_{2}}$, Game $_{\ell_{3}}$, Game $_{\ell_{4}}$, Game $_{\ell_{5}}$, Game $_{\ell_{6}}$, and Game $\ell_{7}$ for $\ell=1, \ldots, Q$. In each game, the first $\ell-1$ keys are semi-functional of type $4 \mathrm{R}$, and all keys after the $\ell$ th request are normal. They differ in the construction of the $\ell$ th key and the ciphertext as follows:

Game $_{\ell_{1}}$ In this game, the $\ell$ th key is type $1 \mathrm{Z}$ and the ciphertext is type 1.

Game $_{\ell_{2}}$ In this game, the $\ell$ th key is type $2 \mathrm{Z}$ and the ciphertext is type 2 .

Game $_{\ell_{3}}$ In this game, the $\ell$ th key is type $3 \mathrm{Z}$ and the ciphertext is type 3.

Game $_{\ell_{4}}$ In this game, the $\ell$ th key is type $3 \mathrm{R}$ and the ciphertext is type 3.

Game $_{\ell_{5}}$ In this game, the $\ell$ th key is type $2 \mathrm{R}$ and the ciphertext is type 2 .

Game $_{\ell_{6}}$ In this game, the $\ell$ th key is type $1 \mathrm{R}$ and the ciphertext is type 1.

Game $_{\ell_{7}}$ In this game, the $\ell$ th key is type $4 \mathrm{R}$ and the ciphertext is type 1.

Note that under this definition, we have that in $\mathbf{G a m e}_{0_{7}}$, the ciphertext given to the attacker is type 1 and the keys are all normal.

The outer structure of our hybrid argument will progress as follows. First, we transition from Game $_{\text {real }}$ to $\mathrm{Game}_{0_{7}}$, then to Game $1_{1}$, next to Game $1_{2}$, next to Game $1_{3}$, next to Game $1_{4}$, next to Game $1_{5}$, next to Game $1_{6}$, next to Game $1_{7}$ and then to $\mathrm{Game}_{2_{1}}$ and so on. We then arrive at $\mathrm{Game}_{Q_{7}}$, where the ciphertext is semifunctional of type 1 and all of the keys given to the attacker are semi-functional of type $4 \mathrm{R}$.

We then transition to one last game named Game ${ }_{\text {final }}$ which will complete our proof. Game $_{\text {final }}$ uses a semi-functional ciphertext of a new type: type X, which we will now define:

Type $\mathbf{X}$ The semi-functional ciphertext of Type $\mathrm{X}$ is formed as:

$$
M X, \quad\left\{g_{p}^{s} g_{q}^{\tilde{s}}, \quad g_{p}^{s A_{K}} g_{p}^{t_{K} B_{K}} g_{q}^{\tilde{s} A_{K}} g_{q}^{\tilde{t}_{K} B_{K}}, \quad g_{p}^{t_{K}} g_{q}^{\tilde{t}_{K}}:(\forall K \in S)\right\}
$$

where $X$ is a uniform random element of $G_{T}$. (again, here $A_{K}=\sum_{j \in K} a_{j}$ and $B_{K}=\sum_{j \in K} b_{j}$ )

Game $_{\text {final }}$ In this game, all keys are semi-functional of type 4R and the ciphertext is semifunctional of type X.

Note that a ciphertext of type $\mathrm{X}$ information-theoretically hides its message $M$ because the message is multiplied by the uniform random $X$ which is unused anywhere else. So, in Game $_{\text {final }}$, no polynomial time adversary will be able to achieve advantage in the security game, completing our proof.

Our hybrid argument is accomplished in the following lemmas:

Lemma 8. Under the Subgroup Decision Assumption 1, no polynomial time attacker can achieve a non-negligible difference in advantage between Game real $_{\text {and }}$ Game $_{0_{7}}$. 
Proof. If an algorithm $\mathcal{A}$ has non-negligible difference in advantage between $\mathrm{Game}_{\text {real }}$ and Game $_{0_{7}}$, then we could use $\mathcal{A}$ to achieve non-negligible advantage in the Subgroup Decision Problem 1 as follows:

Given $g_{p}, g_{w}$ and $T$ where either $T=g_{p}^{s} \leftarrow G_{p}$ or $T=g_{p}^{s} g_{q}^{\tilde{s}} \leftarrow G_{p q}$ (where $s \leftarrow \mathbb{Z}_{p}$ and $\tilde{s} \leftarrow \mathbb{Z}_{q}$ ), consider the following simulator $\mathcal{B}$ in the security game:

The public parameters are formed by using the given $g_{p}$, choosing $\alpha, a_{j}, b_{j}$ randomly from $\mathbb{Z}_{N}$ and giving the constructed public parameters to $\mathcal{A}$. $\mathcal{B}$ can respond to key requests by running the usual key generation algorithm to make normal keys because it knows the $M S K$.

To return the challenge ciphertext for a set of attributes $S$, for each $K \in S$, a $t_{K}^{\prime} \leftarrow \mathbb{Z}_{N}$ is drawn, then the following ciphertext is constructed and provided:

$$
M e\left(g_{p}, T\right)^{\alpha}, \quad\left\{T, \quad T^{A_{K}} T^{t_{K}^{\prime} B_{K}}, \quad T^{t_{K}^{\prime}}:(\forall K \in S)\right\}
$$

The simulator is able to honestly follow the rest of the scheme using generators $g_{p}, g_{w}$, so the only difference between its execution distributions depends on the different ciphertexts formed dependent on $T$.

Notice that if $T=g_{p}^{s}$ for $s \leftarrow \mathbb{Z}_{p}$, then the distribution of ciphertexts formed is identical to the honest case (where $t_{K}=t_{K}^{\prime} s$ ), and so the simulator's behavior is exactly that of Game real $_{\text {. }}$

If $T=g_{p}^{s} g_{q}^{\tilde{s}}$ for $s \leftarrow \mathbb{Z}_{p}, \tilde{s} \leftarrow \mathbb{Z}_{q}$, the ciphertext formed is a semi-functional ciphertext of type 1 (where $t_{K}=t_{K}^{\prime} s$ and $\tilde{t}_{K}=t_{K}^{\prime} \tilde{s}$ are independent since the values of $t_{K}^{\prime}$ modulo $p$ and modulo $q$ are indpendent and uniform in $\mathbb{Z}_{p}, \mathbb{Z}_{q}$ respectively by the Chinese Remainder Theorem), so the simulator's behavior is exactly that of $\mathrm{Game}_{0_{7}}$.

Therefore, any adversary with non-negligible difference in advantage between Game real $_{\text {and }}$ Game $_{0_{7}}$ could be used to achieve the same non-negligible advantage in deciding the Subgroup Decision Problem 1. By assumption this is not possible, so such an adversary cannot exist.

Lemma 9. Under the Subgroup Decision Assumption 2, no polynomial time attacker can achieve a non-negligible difference in advantage between Game $_{(\ell-1)_{7}}$ and ${\text { Game } \ell_{1}}_{1}$ for any $\ell$ from 1 to $Q$

Proof. If an algorithm $\mathcal{A}$ has non-negligible difference in advantage between $\operatorname{Game}_{(\ell-1)_{7}}$ and

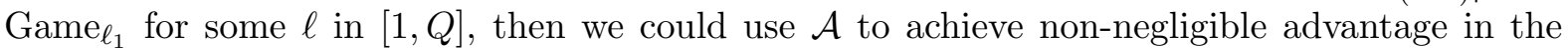
Subgroup Decision Problem 2 as follows:

Given $g_{p}, g_{w}, g_{p}^{s} g_{q}^{\tilde{s}}, g_{q}^{\tilde{r}} g_{w}^{r^{*}}$ and $T$ where either $T=g_{p}^{y} g_{w}^{y^{*}}$ or $T=g_{p}^{y} g_{q}^{\tilde{y}} g_{w}^{y^{*}}$ (where $s, y \leftarrow \mathbb{Z}_{p}$, $\tilde{s}, \tilde{r}, \tilde{y} \leftarrow \mathbb{Z}_{q}$, and $r^{*}, y^{*} \leftarrow \mathbb{Z}_{w}$ ), consider the simulator in the security game which acts as follows:

The public parameters are formed by using the provided $g_{p}$, choosing $\alpha, a_{j}, b_{j}$ randomly from $\mathbb{Z}_{n}$ and giving the constructed public parameters to $\mathcal{A}$.

To return the challenge ciphertext for a set of attributes $S$, for each $K \in S, t_{K}^{\prime} \leftarrow \mathbb{Z}_{N}$ is drawn, then the following semi-functional ciphertext of type 1 is constructed and provided:

$$
M e\left(g_{p}, g_{p}^{s} g_{q}^{\tilde{s}}\right)^{\alpha}, \quad\left\{g_{p}^{s} g_{q}^{\tilde{s}}, \quad\left(g_{p}^{s} g_{q}^{\tilde{s}}\right)^{A_{K}}\left(g_{p}^{s} g_{q}^{\tilde{s}}\right)^{t_{K}^{\prime} B_{K}}, \quad\left(g_{p}^{s} g_{q}^{\tilde{s}}\right)^{t_{K}^{\prime}}:(\forall K \in S)\right\}
$$

Here the semi-functional ciphertext has $t_{K}=\tilde{s} t_{K}^{\prime}$ and $\tilde{t}_{K}=\tilde{s} t_{K}^{\prime}$ which are independent since the values of $t_{K}^{\prime}$ modulo $p$ and modulo $q$ are indpendent and uniform in $\mathbb{Z}_{p}, \mathbb{Z}_{q}$ respectively by the Chinese Remainder Theorem.

For each requested key for policy $\Lambda$, since the simulator knows the MSK $\alpha$, it can generate an honest key:

$$
S K=\left\{g_{p}^{\lambda_{K}} g_{p}^{y_{K} A_{K}} g_{w}^{z_{K}}, \quad g_{p}^{y_{K}} g_{w}^{z_{K}^{\prime}}, \quad g_{p}^{y_{K} B_{K}} g_{w}^{z_{K}^{\prime \prime}}:(\forall K \text { labels } \in \Lambda)\right\}
$$


using the key generation algorithm with $g_{p}$ and $g_{w}$. For honest keys after the $\ell$ th request, the simulator returns this key.

The simulator additionally chooses and fixes $u^{\prime} \leftarrow \mathbb{Z}_{N}$. For key requests up to the $(\ell-1)$ th request, the simulator generates shares $\tilde{\lambda}_{K}^{\prime}$ of $u^{\prime}$. The first $\ell-1$ semi-functional keys of type $4 \mathrm{R}$ can be created by returning:

$$
S K=\left\{g_{p}^{\lambda_{K}} g_{p}^{y_{K} A_{K}}\left(g_{q}^{\tilde{r}} g_{w}^{r^{*}}\right)^{\tilde{\lambda}_{K}^{\prime}} g_{w}^{z_{K}}, \quad g_{p}^{y_{K}} g_{w}^{z_{K}^{\prime}}, \quad g_{p}^{y_{K} B_{K}} g_{w}^{z_{K}^{\prime \prime}}:(\forall K \text { labels } \in \Lambda)\right\}
$$

Note that these are valid semi-functional keys of type $4 \mathrm{R}$ where the $\tilde{\lambda}_{K}=\tilde{r} \tilde{\lambda}_{K}^{\prime}\left(\tilde{\lambda}_{K}^{\prime}\right.$ are a sharing of $u^{\prime}$ so the $\tilde{r} \tilde{\lambda}_{K}^{\prime}$ are a sharing of $u=\tilde{r} u^{\prime}$, which is uniformly distributed in $\mathbb{Z}_{q}$ and fixed across all R-type keys). Also, notice that the extra $G_{w}$ component $g_{w}^{r^{*} \tilde{\lambda}_{K}^{\prime}}$ is absorbed by the uniform random $g_{w}^{z_{K}}$.

For the $\ell$ th key request, the simulator generates $\lambda_{K}^{\prime}$ and $\lambda_{K}^{\prime \prime}$, shares of $\alpha$ and 0 respectively. It then draws $y_{K}^{\prime} \leftarrow \mathbb{Z}_{N}$ and produces the key:

$$
\left\{g_{p}^{\lambda_{K}^{\prime}} T^{\lambda_{K}^{\prime \prime}}\left(T^{y_{K}^{\prime}}\right)^{A_{K}} g_{w}^{z_{K}}, \quad T^{y_{K}^{\prime}} g_{w}^{z_{K}^{\prime}}, \quad\left(T^{y_{K}^{\prime}}\right)^{B_{K}} g_{w}^{z_{K}^{\prime \prime}}:(\forall K \text { labels } \in \Lambda)\right\}
$$

If $T=g_{p}^{y} g_{w}^{y^{*}}$, then the key is an honest key, where $\lambda_{K}=\lambda_{K}^{\prime}+y \lambda_{K}^{\prime \prime}$, which is a valid sharing of $\alpha$ ( $\lambda_{K}^{\prime \prime}$ is a sharing of zero which multiplying it by $y$ does not change, and adding this to $\lambda_{K}^{\prime}$ (the sharing of $\alpha$ ) results in another sharing of $\alpha$ ), and $y_{K}=y_{K}^{\prime} y$. The extra $G_{w}$ components are all absorbed by the uniform random $g_{w}^{z_{K}}, g_{w}^{z_{K}^{\prime}}$, and $g_{w}^{z_{K}^{\prime \prime}}$.

If $T=g_{p}^{y} g_{q}^{\tilde{y}} g_{w}^{y^{*}}$, then the key is semi-functional of type $1 \mathrm{Z}$, where $\lambda_{K}=\lambda_{K}^{\prime}+y \lambda_{K}^{\prime \prime}$, which is again a valid sharing of $\alpha, \tilde{y}_{K}=y_{K}^{\prime} \tilde{y}$, and $y_{K}=y_{K}^{\prime} y$ which are independent since the values of $y_{K}^{\prime}$ modulo $p$ and modulo $q$ are indpendent and uniform in $\mathbb{Z}_{p}, \mathbb{Z}_{q}$ respectively by the Chinese Remainder Theorem. The extra $G_{w}$ components are all absorbed by the uniform random $g_{w}^{z_{K}}, g_{w}^{z_{K}^{\prime}}$, and $g_{w}^{z_{K}^{\prime \prime}}$.

Therefore, any adversary able to achieve a non-negligible difference in advantage between Game $_{(\ell-1)_{7}}$ and Game $_{\ell_{1}}$ for some $\ell$ in $[1, Q]$ could be used to achieve the same non-negligible advantage in deciding the Subgroup Decision Problem 2. By assumption this is not possible, so such an adversary cannot exist.

Bilinear Entropy Expansion Before the next step of the hybrid proof, we will need a result about the indistinguishability between two distributions, based on the 2-Linear Computational Hardness Assumption.

Definition 10. Given $G$, a group of prime order $q$, and $g$ a generator of that group, let $\mathcal{D}_{1}(m)$ be the distribution of:

$$
\begin{aligned}
& g^{\tilde{s}}, \\
& g^{\tilde{y}_{1}}, \ldots, g^{\tilde{y}_{M-1}}, \\
& g^{\tilde{y}_{1} r_{1}}, \ldots, g^{\tilde{y}_{M-1} r_{M-1}}, \\
& g^{\tilde{y}_{1} b_{1}}, \ldots, g^{\tilde{y}_{M-1} b_{M-1}}, \\
& g^{\tilde{t}_{1}}, \ldots, g^{\tilde{t}_{M-1}}, \\
& g^{\tilde{s} r_{1}+\tilde{t}_{1} b_{1}}, \ldots, g^{\tilde{s} r_{M-1}+\tilde{t}_{M-1} b_{M-1}},
\end{aligned}
$$

where the $\tilde{y}_{i}, \tilde{t}_{i}, b_{i}, r_{i}, \tilde{s} \leftarrow \mathbb{Z}_{q}$ and $M=2^{m}$. 
Definition 11. Given $G$, a group of prime order $q, g$ a generator of that group, and $C=$ $\left\{c_{1}, \ldots, c_{m}\right\}$ a set of $m$ elements drawn uniformly at random from $\mathbb{Z}_{q}$, let $\mathcal{D}_{2}(m)$ be the same distribution as above where the $\tilde{y}_{i}, \tilde{t}_{i}, b_{i}, \tilde{s} \leftarrow \mathbb{Z}_{q}$ but each $r_{i}=\sum_{j \in C_{i}} c_{j}$ where $C_{i}$ denotes the $i$ th indexed nonempty subset of $C\left(|C|=m\right.$ and there are $M-1=2^{m}-1$ nonempty subsets).

The Bilinear Entropy Expansion Lemma states that these two distributions are indistinguishable:

Lemma 12. The distributions $\mathcal{D}_{1}(k)$ and $\mathcal{D}_{2}(k)$ are computationally indistinguishable under the 2-Linear computational hardness assumption if $k=O(\lg$ poly $(\lambda))$.

We defer the proof of this lemma to the end of this security proof, and return to the next step in our hybrid: where the $B_{K}$ in the semi-functional space change to random elements $g_{q}^{\tilde{b}_{K}}$.

Lemma 13. Under the 2-Linear Assumption, no polynomial time attacker can achieve a nonnegligible difference in advantage between Game $_{\ell_{1}}$ and $G_{a m e} \ell_{2}$ for any $\ell$ from 1 to $Q$.

Proof. From Lemma 12 we have that the distributions $\mathcal{D}_{1}(k)$ and $\mathcal{D}_{2}(k)$ are computationally indistinguishable under the 2-Linear computational hardness assumption if $k=O(\lg \operatorname{poly}(\lambda))$. However, if an algorithm $\mathcal{A}$ is able to achieve a non-negligible difference in advantage $\delta$ between Game $_{\ell_{1}}$ and Game $_{\ell_{2}}$ for some $\ell$ in $[1, Q]$, then we could use $\mathcal{A}$ to create a distinguisher $\mathcal{B}$ for $\mathcal{D}_{1}(k)$ and $\mathcal{D}_{2}(k)$ where $k$ is the same value that defines our attribute universe $\mathcal{U}$. (This presents a contradiction since $|\mathcal{U}|=O(\operatorname{poly}(\lambda))$ and $\mathcal{U}$ is defined as containing all subsets of $[k]$, so $k=O(\lg$ poly $(\lambda)))$. We do this as follows:

Let the generator of $G_{q}$ used in $\mathcal{D}_{1}(k)$ and $\mathcal{D}_{2}(k)$ be $g_{q}$. Rename the set $C$ as $C=\left\{b_{1}^{\prime}, \ldots, b_{k}^{\prime}\right\}$. Now, given the challenge instance

$$
\begin{aligned}
& g_{q}^{\tilde{s}}, \\
& g_{q}^{\tilde{y}_{1}}, \ldots, g_{q}^{\tilde{y}_{K-1}}, \\
& g_{q}^{\tilde{y}_{1} r_{1}}, \ldots, g_{q}^{\tilde{y}_{K-1} r_{K-1}}, \\
& g_{q}^{\tilde{y}_{1} b_{1}}, \ldots, g_{q}^{\tilde{y}_{K-1} b_{K-1}}, \\
& g_{q}^{\tilde{t}_{1}}, \ldots, g_{q}^{\tilde{t}_{K-1}}, \\
& g_{q}^{\tilde{s} r_{1}+\tilde{t}_{1} b_{1}}, \ldots, g_{q}^{\tilde{s} r_{K-1}+\tilde{t}_{K-1} b_{K-1}}
\end{aligned}
$$

relabel each index as the subset $K \subseteq[k]$ associated to it, so in particular we have:

$$
g_{q}^{\tilde{y}_{K}}, g_{q}^{\tilde{y}_{K} r_{K}}
$$

for all nonempty subsets $K \subseteq[k]$. (Note that here we do not even use all of the challenge instance, just the sections of $g_{q}^{\tilde{y}_{K}}, g_{q}^{\tilde{y}_{K} r_{K}}$. The full set is needed later when we reuse the lemma in the next hybrid proof).

Now, $\mathcal{B}$ forms the public parameters using $g_{p}$, choosing $\alpha, a_{j}, b_{j}$ randomly and giving the appropriately constructed public parameters to $\mathcal{A}$.

To return the challenge ciphertext for a set of attributes $S$, first choose an $s \leftarrow \mathbb{Z}_{N}$. The simulator then redefines $\tilde{s}$ by sampling it uniformly at random from $\mathbb{Z}_{N}$. Then, $t_{K}, t_{K}^{*} \leftarrow \mathbb{Z}_{N}$ are chosen for each $K \in S$. The following semi-functional ciphertext can then be constructed and provided:

$$
M e\left(g_{p}, g_{p}\right)^{\alpha s}, \quad\left\{g_{p}^{s} g_{q}^{\tilde{s}}, \quad g_{p}^{s A_{K}} g_{p}^{t_{K} B_{K}} g_{q}^{\tilde{s} A_{K}}\left(g_{q}^{\tilde{y}_{K} r_{K}}\right)^{t_{K}^{*}}, \quad g_{p}^{t_{K}}\left(g_{q}^{\tilde{y}_{K}}\right)^{t_{K}^{*}}:(\forall K \in S)\right\}
$$


Here, $\tilde{t}_{K}=t_{K}^{*} \tilde{y}_{K}$ for all $K \in S$.

For each requested key $S$ for policy $\Lambda$, since the simulator knows the MSK $\alpha$, it can generate an honest key:

$$
S K=\left\{g_{p}^{\lambda_{K}} g_{p}^{y_{K} A_{K}} g_{w}^{z_{K}}, \quad g_{p}^{y_{K}} g_{w}^{z_{K}^{\prime}}, \quad g_{p}^{y_{K} B_{K}} g_{w}^{z_{K}^{\prime \prime}}:(\forall K \text { labels } \in \Lambda)\right\}
$$

using the key generation algorithm with $g_{p}$ and $g_{w}$. For honest keys after the $\ell$ th request, the simulator returns this key.

The simulator additionally chooses and fixes $u \leftarrow \mathbb{Z}_{N}$. For key requests up to the $(\ell-1)$ th request, the simulator generates shares $\tilde{\lambda}_{K}$ of $u$. The first $\ell-1$ semi-functional keys of type $4 \mathrm{R}$ can be created by returning:

$$
S K=\left\{g_{p}^{\lambda_{K}} g_{p}^{y_{K} A_{K}} g_{q}^{\tilde{\lambda}_{K}} g_{w}^{z_{K}}, \quad g_{p}^{y_{K}} g_{w}^{z_{K}^{\prime}}, \quad g_{p}^{y_{K} B_{K}} g_{w}^{z_{K}^{\prime \prime}}:(\forall K \text { labels } \in \Lambda)\right\}
$$

For the $\ell$ th key request, $\mathcal{B}$ can compute $\tilde{\lambda}_{K}$ : a sharing of 0 and return the key:

$$
\left\{g_{p}^{\lambda_{K}} g_{p}^{y_{K} A_{K}} g_{q}^{\tilde{\lambda}_{K}}\left(g_{q}^{\tilde{y}_{K}}\right)^{A_{K}} g_{w}^{z_{K}}, \quad g_{p}^{y_{K}}\left(g_{q}^{\tilde{y}_{K}}\right) g_{w}^{z_{K}^{\prime}}, \quad g_{p}^{y_{K} B_{K}}\left(g_{q}^{\tilde{y}_{K} r_{K}}\right) g_{w}^{z_{K}^{\prime \prime}}:(\forall K \text { labels } \in \Lambda)\right\}
$$

Notice that if $r_{K}=\sum_{j \in K} b_{j}^{\prime}$, then $g_{q}^{r_{K}}=\prod_{j \in K} g_{q}^{b_{j}^{\prime}}$ is distributed identically to $g_{q}^{B_{K}}=\prod_{j \in K} g_{q}^{b_{j}}$ by the Chinese Remainder Theorem. Each $b_{j}$ is a random element of $\mathbb{Z}_{N}$ so its value taken $\bmod q$ is independent of its value $\bmod p$. So, the distributions of $\left(\prod_{j \in K} g_{p}^{b_{j}}, \prod_{j \in K} g_{q}^{b_{j}}\right)=\left(g_{p}^{B_{K}}, g_{q}^{B_{K}}\right)$ and $\left(\prod_{j \in K} g_{p}^{b_{j}}, \prod_{j \in K} g_{q}^{b_{j}^{\prime}}\right)=\left(g_{p}^{B_{K}}, g_{q}^{r_{K}}\right)$ are identical.

Constructing the $\ell$ th key and challenge ciphertext this way gives them alternatively structured or random components depending on whether the $r_{K}=\sum_{j \in K} b_{j}^{\prime}$ or are uniformly random in $\mathbb{Z}_{q}$. If the challenge set is drawn from $\mathcal{D}_{2}(k)$, then $\mathcal{B}$ behaves as expected in Game $\ell_{\ell_{1}}$ (where the challenge ciphertext and $\ell$ th key are semi-functional of type 1 and $1 \mathrm{Z}$ respectively). If the challenge set is drawn from $\mathcal{D}_{1}(k)$, then $\mathcal{B}$ behaves as expected in Game $\ell_{2}$ (where the challenge ciphertext and $\ell$ th key are semi-functional of type 2 and $2 \mathrm{Z}$ respectively).

Therefore, any adversary with non-negligible difference in advantage $\delta$ between Game $\ell_{\ell_{1}}$ and Game $_{\ell_{2}}$ could be used to achieve the same non-negligible difference in advantage in distinguishing the distributions $\mathcal{D}_{1}(k)$ and $\mathcal{D}_{2}(k)$. By Lemma 12 this is not possible, so such an adversary cannot exist and therefore we have proven that no polynomial time attacker can achieve a non-negligible difference in advantage between Game $\ell_{1}$ and Game $\ell_{2}$ for any $\ell$ from 1 to $Q$.

The proof for the next step in our hybrid is similar, as we transition from structured $g_{q}^{A_{K}}$ to random elements $g_{q}^{\tilde{a}_{K}}$ (except this uses the full version of our Bilinear Entropy Expansion lemma):

Lemma 14. Under the 2-Linear Assumption, no polynomial time attacker can achieve a nonnegligible difference in advantage between $G_{a m \ell_{\ell_{2}}}$ and $G_{a m e} e_{\ell_{3}}$ for any $\ell$ from 1 to $Q$.

Proof. From Lemma 12 we have that the distributions $\mathcal{D}_{1}(k)$ and $\mathcal{D}_{2}(k)$ are computationally indistinguishable under the 2-Linear computational hardness assumption if $k=O(\lg \operatorname{poly}(\lambda))$. However, if an algorithm $\mathcal{A}$ is able to achieve non-negligible difference in advantage $\delta$ between

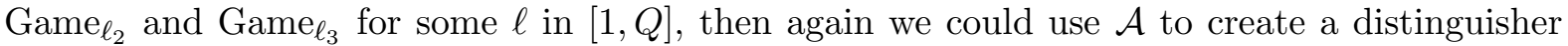
$\mathcal{B}$ for $\mathcal{D}_{1}(k)$ and $\mathcal{D}_{2}(k)$ (where again we use the same $k$ which defines our attribute universe $\mathcal{U}$ and satisfies $k=O(\lg \operatorname{pol} y(\lambda)))$. We do this as follows: 
Let the generator of $G_{q}$ used in $\mathcal{D}_{1}(k)$ and $\mathcal{D}_{2}(k)$ be $g_{q}$. Relabel the set $C$ as $C=\left\{a_{1}^{\prime}, \ldots, a_{k}^{\prime}\right\}$. Now, given the challenge instance

$$
\begin{aligned}
& g_{q}^{\tilde{s}}, \\
& g_{q}^{\tilde{y}_{1}}, \ldots, g_{q}^{\tilde{y}_{K-1}}, \\
& g_{q}^{\tilde{y}_{1} r_{1}}, \ldots, g_{q}^{\tilde{y}_{K-1} r_{K-1}}, \\
& g_{q}^{\tilde{y}_{1} b_{1}}, \ldots, g_{q}^{\tilde{y}_{K-1} b_{K-1}}, \\
& g_{q}^{\tilde{t}_{1}}, \ldots, g_{q}^{\tilde{t}_{K-1}}, \\
& g_{q}^{\tilde{s} r_{1}+\tilde{t}_{1} b_{1}}, \ldots, g_{q}^{\tilde{s} r_{K-1}+\tilde{t}_{K-1} b_{K-1}}
\end{aligned}
$$

relabel each index as the subset $K \subseteq[k]$ associated to it, so we have:

$$
g_{q}^{\tilde{s}}, g_{q}^{\tilde{y}_{K}}, g_{q}^{\tilde{y}_{K} r_{K}}, g_{q}^{\tilde{y}_{K} b_{K}}, g_{q}^{\tilde{t}_{K}}, g_{q}^{\tilde{s}_{K}}+\tilde{t}_{K} b_{K}
$$

for all nonempty subsets $K \subseteq[k]$.

Now, $\mathcal{B}$ forms the public parameters using $g_{p}$, choosing $\alpha, a_{j}, b_{j}$ randomly and giving the appropriately constructed public parameters to $\mathcal{A}$.

To return the challenge ciphertext for a set of attributes $S$, first choose an $s \leftarrow \mathbb{Z}_{N}$. Then, $t_{K} \leftarrow \mathbb{Z}_{N}$ are chosen for all $K \in S$. The following semi-functional ciphertext can then be constructed and provided:

$$
M e\left(g_{p}, g_{p}\right)^{\alpha s}, \quad\left\{g_{p}^{s}\left(g_{q}^{\tilde{s}}\right), \quad g_{p}^{s A_{K}} g_{p}^{t_{K} B_{K}}\left(g_{q}^{\tilde{s} r_{K}+\tilde{t}_{K} b_{K}}\right), \quad g_{p}^{t_{K}} g_{q}^{\tilde{t}_{K}}:(\forall K \in S)\right\}
$$

Note that here we implicitly set $\tilde{b}_{K}=b_{K}$.

For each requested key for policy $\Lambda$, since the simulator knows the MSK $\alpha$, it can generate an honest key:

$$
S K=\left\{g_{p}^{\lambda_{K}} g_{p}^{y_{K} A_{K}} g_{w}^{z_{K}}, \quad g_{p}^{y_{K}} g_{w}^{z_{K}^{\prime}}, \quad g_{p}^{y_{K} B_{K}} g_{w}^{z_{K}^{\prime \prime}}:(\forall K \text { labels } \in \Lambda)\right\}
$$

using the key generation algorithm with $g_{p}$ and $g_{w}$. For honest keys after the $\ell$ th request, the simulator returns this key.

The simulator additionally chooses and fixes $u \leftarrow \mathbb{Z}_{N}$. For key requests up to the $(\ell-1)$ th request, the simulator generates shares $\tilde{\lambda}_{K}$ of $u$. The first $\ell-1$ semi-functional keys of type $4 \mathrm{R}$ can be created by returning:

$$
S K=\left\{g_{p}^{\lambda_{K}} A_{K}^{y_{K}} g_{q}^{\tilde{\lambda}_{K}} g_{w}^{z_{K}}, \quad g_{p}^{y_{K}} g_{w}^{z_{K}^{\prime}}, \quad g_{p}^{y_{K} B_{K}} g_{w}^{z_{K}^{\prime \prime}}:(\forall K \text { labels } \in \Lambda)\right\}
$$

For the $\ell$ th key request, $\mathcal{B}$ can compute $\tilde{\lambda}_{K}$ : a sharing of 0 , and return the key:

$$
\left\{g_{p}^{\lambda_{K}} g_{p}^{y_{K} A_{K}} g_{q}^{\tilde{\lambda}_{K}}\left(g_{q}^{\tilde{y}_{K} r_{K}}\right) g_{w}^{z_{K}}, \quad g_{p}^{y_{K}}\left(g_{q}^{\tilde{y}_{K}}\right) g_{w}^{z_{K}^{\prime}}, \quad g_{p}^{y_{K} B_{K}}\left(g_{q}^{\tilde{y}_{K} b_{K}}\right) g_{w}^{z_{K}^{\prime \prime}}:(\forall K \text { labels } \in \Lambda)\right\}
$$

Again, notice that if $r_{K}=\sum_{j \in K} a_{j}^{\prime}$, then $g_{q}^{A_{K}}=\prod_{j \in K} g_{q}^{a_{j}}$ is distributed identically to $\prod_{j \in K} g_{q}^{a_{j}^{\prime}}=$ $g_{q}^{r_{K}}$ by the Chinese Remainder Theorem. Each $a_{j}$ is a random element of $\mathbb{Z}_{N}$ so its value taken $\bmod q$ is independent of its value $\bmod p$. So, the distributions of $\left(\prod_{j \in K} g_{p}^{a_{j}}, \prod_{j \in K} g_{q}^{a_{j}}\right)=\left(g_{p}^{A_{K}}, g_{q}^{A_{K}}\right)$ and $\left(\prod_{j \in K} g_{p}^{a_{j}}, \prod_{j \in K} g_{q}^{a_{j}^{\prime}}\right)=\left(g_{p}^{A_{K}}, g_{q}^{r_{K}}\right)$ are identical. 
So, constructing the challenge ciphertext and $\ell$ th key this way causes them to have alternatively structured or random components depending on whether the $r_{K}=\sum_{j \in K} a_{j}^{\prime}$ or are uniformly random in $\mathbb{Z}_{q}$. If the challenge set is drawn from $\mathcal{D}_{2}(k)$, then $\mathcal{B}$ behaves as expected in Game $\ell_{2}$ (where the challenge ciphertext and $\ell$ th key are semi-functional of type 2 and $2 \mathrm{Z}$ respectively). If the challenge set is drawn from $\mathcal{D}_{1}(k)$, then $\mathcal{B}$ behaves as expected in $\mathbf{G a m e}_{\ell_{3}}$ (where the challenge ciphertext and $\ell$ th key are semi-functional of type 3 and $3 \mathrm{Z}$ respectively).

Therefore, any adversary with non-negligible difference in advantage $\delta$ between Game $_{\ell_{2}}$ and Game $_{\ell_{3}}$. could be used to achieve the same non-negligible difference in advantage in distinguishing the distributions $\mathcal{D}_{1}(k)$ and $\mathcal{D}_{2}(k)$. By Lemma 12 this is not possible, so such an adversary cannot exist and therefore we have proven that no polynomial time attacker can achieve a non-negligible difference in advantage between Game $_{\ell_{2}}$ and Game $\ell_{3}$ for any $\ell$ from 1 to $Q$.

We continue to the next step in our hybrid proof:

Lemma 15. No polynomial time attacker can achieve a non-negligible difference in advantage between Game $_{\ell_{3}}$ and Game $_{\ell_{4}}$ for any $\ell$ from 1 to $Q$

Proof. Recall that in both $\mathrm{Game}_{\ell_{3}}$ and $\mathrm{Game}_{\ell_{4}}$, the ciphertext is semi-functional of type 3, all keys after the $\ell$ th key request are normal, and the first $\ell-1$ keys are semi-functional of type $4 \mathrm{R}$. The only difference is the $\ell$ th key (either semi-functional of type $3 \mathrm{Z}$ or $3 \mathrm{R}$ ). In Game $\ell_{3}$, the shares $\tilde{\lambda}_{K}$ are a sharing of 0 , while in Game $\ell_{\ell_{4}}$, they are a sharing of the $u$ used in R-type keys. The transition between these two modes of operation is accomplished using an informationtheoretic argument. Namely, the distribution of elements seen by an attacker in both games is the same.

To see this, note that for any attribute $K$ not included in the ciphertext, then the distributions of the elements of the key indexed by $K$ are identical in both cases, since the $\tilde{\lambda}_{K}$ are masked by a $g_{q}^{\tilde{a}_{K}} \tilde{y}_{K}$ where the $\tilde{a}_{K}$ are chosen uniformly at random and used nowhere else (they do not appear in keys other than the $\ell$ th key and only appear at most once in the $\ell$ th key because of our single-use restriction), therefore hiding the value of all $\tilde{\lambda}_{K}$ information-theoretically.

For attributes $K$ used in the ciphertext, this argument does not apply (since these $\tilde{a}_{K}$ do appear elsewhere - in the ciphertext). For these, note that in the security game the attacker is not allowed to request a key that is able to decrypt the challenge ciphertext. So, for this key with policy $\Lambda$, the rows of the policy matrix $\Lambda$ corresponding to the attributes the challenge ciphertext is encrypted under do not contain $(1,0, \ldots, 0)$ in their span (modulo $N$ ). We can further assume that the corresponding rows of $\Lambda$ do not include $(1,0, \ldots, 0)$ in their span modulo $q$ (because if an adversary can produce an unauthorized set over $\mathbb{Z}_{N}$ that is authorized over $\mathbb{Z}_{q}$, then this can be used to produce a non-trivial factor of the group order $N$, which would violate our subgroup decision assumptions). Therefore, there exists some vector $\vec{w} \in \mathbb{Z}_{q}^{n}$ orthogonal to the span of these rows which is not orthogonal to $(1,0, \ldots, 0)$ modulo $q$ (since $\mathbb{Z}_{q}$ is a finite field). By scaling this vector we can have $\vec{w}=\left(1, w_{2}, \ldots, w_{n}\right)$ for some collection of $w_{i}$. Now notice that whether the shares $\tilde{\lambda}_{K}$ which comprise $\overrightarrow{\tilde{\lambda}}$ were generated by taking $\Lambda \vec{r}=\overrightarrow{\tilde{\lambda}}$ where $\vec{r}=\left(0, r_{2}, \ldots, r_{n}\right)$ (that is, a valid sharing of zero) or taking $\Lambda(\vec{r}+u \vec{w})$ where (that is, a valid sharing of the $u$ used in R-type keys), then the distributions of shares $\tilde{\lambda}_{K}$ modulo $q$ (they are exponents of $g_{q}$ ) that are not information-theoretically hidden by the previous argument are the same. All that is seen are $\tilde{\lambda}_{K}$ created by taking dot products with rows of $\Lambda$ - for the shares that are not information-theoretically hidden, we have that the $u \vec{w}$ contributes 0 modulo $q$ to the share so the distribution of these shares is the same as those produced by an honest sharing of zero. 
Since the distributions of elements of the key given the ciphertext are identical whether the $\tilde{\lambda}_{K}$ are formed by taking a sharing of zero or a random element of $\mathbb{Z}_{N}$, then no algorithm can tell the difference between the two games, and so we have proven the lemma.

Now that we have changed the $\tilde{\lambda}_{K}$ from sharing 0 to sharing $u$, we proceed to restructure the $A_{K}, B_{K}$ :

Lemma 16. Under the 2-Linear Assumption, no polynomial time attacker can achieve a nonnegligible difference in advantage between $G_{a m \ell_{\ell_{4}}}$ and Game $_{\ell_{5}}$ for any $\ell$ from 1 to $Q$.

Proof. Note that this proof is identical to Lemma 14, (which uses the Bilinear Entropy Expansion Lemma 12), with the only difference being that now the $\tilde{\lambda}_{K}$ are a sharing of a random element of $\mathbb{Z}_{N}$ instead of 0 . So, the only change in the proof is that instead of generating $\tilde{\lambda}_{K}$ as a sharing of 0 , the $\tilde{\lambda}_{K}$ are generated as a sharing of $u$ : a fixed random element of $\mathbb{Z}_{N}$. The simulator is equally capable of making either set of shares, and using the sharing of a random element does not affect the simulator's ability to perform any of the other actions detailed in the remainder of Lemma 14.

Lemma 17. Under the 2-Linear Assumption, no polynomial time attacker can achieve a nonnegligible difference in advantage between $G_{a m e} \ell_{5}$ and $G_{a m e} \ell_{6}$ for any $\ell$ from 1 to $Q$.

Proof. Note that this proof is again identical to Lemma 13, with the only difference being that now the $\tilde{\lambda}_{K}$ are a sharing of a random element of $\mathbb{Z}_{N}$ instead of 0 . So, again, the only change in the proof is that instead of generating $\tilde{\lambda}_{K}$ as a sharing of 0 , the $\tilde{\lambda}_{K}$ are generated as a sharing of $u$ : a fixed random element of $\mathbb{Z}_{N}$. The simulator is equally capable of making either set of shares, and using the sharing of a random element does not affect the simulator's ability to perform any of the other actions detailed in the remainder of Lemma 13.

Lemma 18. Under the Subgroup Decision Assumption 2, no polynomial time attacker can achieve a non-negligible difference in advantage between ${\text { Game } \ell_{6}}$ and $G_{a m e} e_{7}$ for any $\ell$ from 1 to $Q$

Proof. If an algorithm $\mathcal{A}$ achieves non-negligible difference in advantage between Game $\ell_{6}$ and

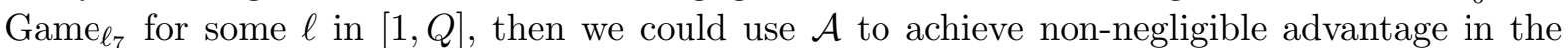
Subgroup Decision Problem 2 as follows:

Given $g_{p}, g_{w}, g_{p}^{s} g_{q}^{\tilde{s}}, g_{q}^{\tilde{r}} g_{w}^{r^{*}}$ and $T$ where either $T=g_{p}^{y} g_{w}^{y^{*}}$ or $T=g_{p}^{y} g_{q}^{\tilde{y}} g_{w}^{y^{*}}$ (where $s, y \leftarrow \mathbb{Z}_{p}$, $\tilde{s}, \tilde{r}, \tilde{y} \leftarrow \mathbb{Z}_{q}$, and $r^{*}, y^{*} \leftarrow \mathbb{Z}_{w}$ ), consider the simulator in the security game which acts as follows:

The public parameters are formed by using the provided $g_{p}$, choosing $\alpha, a_{j}, b_{j}$ randomly and giving the constructed public parameters to $\mathcal{A}$.

To return the challenge ciphertext for a set of attributes $S$, for each $K \in S$, a $t_{K}^{\prime} \leftarrow \mathbb{Z}_{N}$ is chosen, then the following semi-functional ciphertext of type 1 is constructed and provided:

$$
M e\left(g_{p}, g_{p}^{s} g_{q}^{\tilde{s}}\right)^{\alpha}, \quad\left\{g_{p}^{s} g_{q}^{\tilde{s}}, \quad\left(g_{p}^{s} g_{q}^{\tilde{s}}\right)^{A_{K}}\left(g_{p}^{s} g_{q}^{\tilde{s}}\right)^{t_{K}^{\prime} B_{K}}, \quad\left(g_{p}^{s} g_{q}^{\tilde{s}}\right)^{t_{K}^{\prime}}:(\forall K \in S)\right\}
$$

Here the semi-functional ciphertext has $t_{K}=\tilde{s} t_{K}^{\prime}$ and $\tilde{t}_{K}=\tilde{s} t_{K}^{\prime}$ which are independent since the values of $t_{K}^{\prime}$ modulo $p$ and modulo $q$ are independent and uniform in $\mathbb{Z}_{p}, \mathbb{Z}_{q}$ respectively by the Chinese Remainder Theorem.

For each requested key for policy $\Lambda$, since the simulator knows the MSK $\alpha$, it can generate an honest key:

$$
S K=\left\{g_{p}^{\lambda_{K}} g_{p}^{y_{K} A_{K}} g_{w}^{z_{K}}, \quad g_{p}^{y_{K}} g_{w}^{z_{K}^{\prime}}, \quad g_{p}^{y_{K} B_{K}} g_{w}^{z_{K}^{\prime \prime}}:(\forall K \text { labels } \in \Lambda)\right\}
$$


using the key generation algorithm with $g_{p}$ and $g_{w}$. For honest keys after the $\ell$ th request, the simulator returns this key. The first $\ell-1$ semi-functional keys of type $4 \mathrm{R}$ can be created by generating shares $\tilde{\lambda}_{K}^{\prime}$ of a fixed $u^{\prime} \leftarrow \mathbb{Z}_{q}$ and returning:

$$
S K=\left\{g_{p}^{\lambda_{K}} g_{p}^{y_{K} A_{K}}\left(g_{q}^{\tilde{r}} g_{w}^{r^{*}}\right)^{\tilde{\lambda}_{K}^{\prime}} g_{w}^{z_{K}}, \quad g_{p}^{y_{K}} g_{w}^{z_{K}^{\prime}}, \quad g_{p}^{y_{K} B_{K}} g_{w}^{z_{K}^{\prime \prime}}:(\forall K \text { labels } \in \Lambda)\right\}
$$

Note that these are valid semi-functional keys of type $4 \mathrm{R}$ where the $\tilde{\lambda}_{K}=\tilde{r} \tilde{\lambda}_{K}^{\prime}$ and the extra $G_{w}$ component is absorbed by the uniform random $g_{w}^{z_{K}}$.

For the $\ell$ th key request, the simulator generates $\lambda_{K}$ and $\tilde{\lambda}_{K}^{\prime}$, shares of $\alpha$ and $u^{\prime}$ respectively. It then draws $y_{K}^{\prime}$ uniformly at random from $\mathbb{Z}_{N}$ and produces the key:

$$
\left\{g_{p}^{\lambda_{K}}\left(g_{q}^{\tilde{r}} g_{w}^{r^{*}}\right)^{\tilde{\lambda}_{K}^{\prime}}\left(T^{y_{K}^{\prime}}\right)^{A_{K}} g_{w}^{z_{K}}, \quad T^{y_{K}^{\prime}} g_{w}^{z_{K}^{\prime}}, \quad\left(T^{y_{K}^{\prime}}\right)^{B_{K}} g_{w}^{z_{K}^{\prime \prime}}:(\forall K \text { labels } \in \Lambda)\right\}
$$

Again, if $\tilde{\lambda}_{K}^{\prime}$ are a sharing of $u^{\prime}$, then $\tilde{r} \tilde{\lambda}_{K}^{\prime}$ is the sharing of $\tilde{r} u^{\prime}$, which is fixed across all R-type keys.

If $T=g_{p}^{y} g_{q}^{\tilde{y}} g_{w}^{y^{*}}$, then the key is semi-functional of type $1 \mathrm{R}$, where $\tilde{y}_{K}=y_{K}^{\prime} \tilde{y}$, and $y_{K}=y_{K}^{\prime} y$ which are independent since the values of $y_{K}^{\prime}$ modulo $p$ and modulo $q$ are independent and uniform in $\mathbb{Z}_{p}, \mathbb{Z}_{q}$ respectively by the Chinese Remainder Theorem. The extra $G_{w}$ components are all absorbed by the uniform random $g_{w}^{z_{K}}, g_{w}^{z_{K}^{\prime}}$, and $g_{w}^{z_{K}^{\prime \prime}}$.

If $T=g_{p}^{y} g_{w}^{y^{*}}$, then the key is a semi-functional key of type $4 \mathrm{R}$, where $y_{K}=y_{K}^{\prime} y$. The extra $G_{w}$ components are all absorbed by the uniform random $g_{w}^{z_{K}}, g_{w}^{z_{K}^{\prime}}$, and $g_{w}^{z_{K}^{\prime \prime}}$.

Therefore, any adversary able to achieve a non-negligible difference in advantage between ${\text { Game } \ell_{6}}_{\text {and }}$ Game $_{\ell_{7}}$ for some $\ell$ in $[1, Q]$ could be used to achieve the same non-negligible advantage in deciding the Subgroup Decision Problem 2. By assumption this is not possible, so such an adversary cannot exist.

This set of hybrids takes us to Game $Q_{7}$ : where the semi-functional ciphertext is of type 1 and all keys are semi-functional of type $4 \mathrm{R}$. We take one more step to Game final where the distribution of the ciphertext is independent of the message - a game in which the adversary cannot have any advantage:

Lemma 19. Under the Subgroup Decision Assumption 3, no polynomial time attacker can achieve a non-negligible difference in advantage between Game $_{Q_{7}}$ and Game final $_{\text {. }}$

Proof. If an algorithm $\mathcal{A}$ is able to achieve a non-negligible difference in advantage between Game $_{Q_{7}}$ and Game $_{\text {final }}$, then we could use $\mathcal{A}$ to break the Subgroup Decision Assumption as follows:

Given $g_{p}, g_{q}, g_{w}, g_{p}^{\alpha} g_{q}^{\tilde{\alpha}}, g_{p}^{s} g_{q}^{\tilde{s}}$, and $T$ where either $T=e\left(g_{p}, g_{p}\right)^{\alpha s}$ or $X$ (where $\alpha, s \leftarrow \mathbb{Z}_{p}$, $\tilde{\alpha}, \tilde{s} \leftarrow \mathbb{Z}_{q}$, and $X \leftarrow G_{T}$ ), consider the simulator in the security game which acts as follows:

The public parameters are formed by using the provided $g_{p}$, computing $e\left(g_{p}, g_{p}^{\alpha} g_{q}^{\tilde{\alpha}}\right)=$ $e\left(g_{p}, g_{p}\right)^{\alpha}$, choosing $a_{j}, b_{j}$ randomly and giving the constructed public parameters to $\mathcal{A}$.

To return the challenge ciphertext for a set of attributes $S$, for each $K \in S, t_{K}, \tilde{t}_{K} \leftarrow \mathbb{Z}_{N}$ are chosen, then the following ciphertext is constructed and provided:

$$
M T, \quad\left\{\left(g_{p}^{s} g_{q}^{\tilde{s}}\right), \quad\left(g_{p}^{s} g_{q}^{\tilde{s}}\right)^{A_{K}} g_{p}^{t_{K} B_{K}} g_{q}^{\tilde{t}_{K} B_{K}}, \quad g_{p}^{t_{K}} g_{q}^{\tilde{t}_{K}}:(\forall K \in S)\right\}
$$

For all key requests, note that the simulator no longer has the value $\alpha$ (only $g_{p}^{\alpha} g_{q}^{\tilde{\alpha}}$ ). However, note that it can still generate $g_{p}^{\lambda_{K}} g_{q}^{\tilde{\lambda}_{K}}$ where the $\lambda_{K}$ are a sharing of $\alpha$ and $\tilde{\lambda}_{K}$ are a sharing 
of $\tilde{\alpha}$ by performing the same share-generation procedure starting with $g_{p}^{\alpha} g_{q}^{\tilde{\alpha}}$ in parallel for both subgroups, just in the exponent of $g_{p} g_{q}$ (each of which it has, separately), by choosing random $r_{i}, \tilde{r}_{i}$, computing $g_{p}^{r_{i}} g_{q}^{\tilde{r}_{i}}$, and raising to appropriate exponents from the policy matrix / computing products to get the desired dot product in the exponent. It then draws $y_{K} \leftarrow \mathbb{Z}_{N}$ and $g_{w}^{z_{K}}, g_{w}^{z_{K}^{\prime}}, g_{w}^{z_{K}^{\prime \prime}}$ uniformly at random from $G_{w}$ (by choosing exponents of $g_{w}$ uniformly at random from $\mathbb{Z}_{n}$ ), finally producing the semi-functional key of type $4 \mathrm{R}$ (where $u=\tilde{\alpha}$ ):

$$
\left\{g_{p}^{\lambda_{K}} g_{q}^{\tilde{\lambda}_{K}} g_{p}^{y_{K} A_{K}} g_{w}^{z_{K}}, \quad g_{p}^{y_{K}} g_{w}^{z_{K}^{\prime}}, \quad g_{p}^{y_{K} B_{K}} g_{w}^{z_{K}^{\prime \prime}}:(\forall K \text { labels } \in \Lambda)\right\}
$$

Note that when $T=e\left(g_{p}, g_{p}\right)^{\alpha s}$, then the simulator is acting exactly as in Game $Q_{7}$ (where the ciphertext is distributed as a semi-functional ciphertext of type 1). When $T=X$ (a completely random element of $G_{T}$ ), then the ciphertext is distributed as a semi-functional ciphertext of type $\mathrm{X}$ (as in $\mathrm{Game}_{\text {final }}$ ) and the message is information-theoretically masked.

So, any adversary able to achieve a non-negligible difference in advantage between Game $_{Q_{7}}$ and Game $_{\text {final }}$ could be used to achieve the same non-negligible advantage in deciding the Subgroup Decision Problem 3. By assumption this is not possible, so such an adversary cannot exist.

We have now proven the following theorem

Theorem 20. Under the 2-Linear Computational Hardness Assumption, Subgroup Decision Assumption 1, Subgroup Decision Assumption 2, and Subgroup Decision Assumption 3, our single-use KP-ABE scheme is fully secure.

Proof. If the 2-Linear Computational Hardness Assumption, Subgroup Decision Assumption 1, Subgroup Decision Assumption 2, and Subgroup Decision Assumption 3 hold, then by the previous lemmas, we have shown that the real security game is computationally indistinguishable from $\mathrm{Game}_{\text {final }}$, in which the challenge ciphertext's message is information-theoretically hidden from the attacker. Hence, no attacker can achieve a non-negligible advantage in breaking the KP-ABE scheme.

\section{Bilinear Entropy Expansion Lemma}

We return to the deferred distribution indistinguishability proof. Recall the definitions of the two distributions:

Definition 21. Given $G$, a group of prime order $q$, and $g$ a generator of that group, let $\mathcal{D}_{1}(m)$ be the distribution of:

$$
\begin{aligned}
& g^{\tilde{s}}, \\
& g^{\tilde{y}_{1}}, \ldots, g^{\tilde{y}_{M-1}}, \\
& g^{\tilde{y}_{1} r_{1}}, \ldots, g^{\tilde{y}_{M-1} r_{M-1}}, \\
& g^{\tilde{y}_{1} b_{1}}, \ldots, g^{\tilde{y}_{M-1} b_{M-1}}, \\
& g^{\tilde{t}_{1}}, \ldots, g^{\tilde{t}_{M-1}}, \\
& g^{\tilde{s} r_{1}+\tilde{t}_{1} b_{1}}, \ldots, g^{\tilde{s} r_{M-1}+\tilde{t}_{M-1} b_{M-1}},
\end{aligned}
$$

where the $\tilde{y}_{i}, \tilde{t}_{i}, b_{i}, r_{i}, \tilde{s} \leftarrow \mathbb{Z}_{q}$ and $M=2^{m}$. 
Definition 22. Given $G$, a group of prime order $q, g$ a generator of that group, and $C=$ $\left\{c_{1}, \ldots, c_{m}\right\}$ a set of $m$ elements drawn uniformly at random from $\mathbb{Z}_{q}$, let $\mathcal{D}_{2}(m)$ be the distribution of:

$$
\begin{aligned}
& g^{\tilde{s}}, \\
& g^{\tilde{y}_{1}}, \ldots, g^{\tilde{y}_{M-1}}, \\
& g^{\tilde{y}_{1} r_{1}}, \ldots, g^{\tilde{y}_{M-1} r_{M-1}}, \\
& g^{\tilde{y}_{1} b_{1}}, \ldots, g^{\tilde{y}_{M-1} b_{M-1}}, \\
& g^{\tilde{t}_{1}}, \ldots, g^{\tilde{t}_{M-1}}, \\
& g^{\tilde{s}_{1}+\tilde{t}_{1} b_{1}}, \ldots, g^{\tilde{s} r_{M-1}+\tilde{t}_{M-1} b_{M-1}},
\end{aligned}
$$

where the $\tilde{y}_{i}, \tilde{t}_{i}, b_{i}, \tilde{s} \leftarrow \mathbb{Z}_{q}$ and $M=2^{m}$ but each $r_{i}=\sum_{j \in C_{i}} c_{j}$ where $C_{i}$ denotes the $i$ th indexed nonempty subset of $C\left(|C|=m\right.$ and there are $M-1=2^{m}-1$ nonempty subsets).

We show that the distributions $\mathcal{D}_{1}(m)$ and $\mathcal{D}_{2}(m)$ are computationally indistinguishable if $m=O(\lg$ poly $(\lambda))$ through an inductive proof, beginning with the base case of $m=2$, where a distinguisher for $\mathcal{D}_{1}(2)$ and $\mathcal{D}_{2}(2)\left(C=\left\{c_{1}, c_{2}\right\}\right)$ can be used to achieve the same advantage in the 2-Linear Problem.

Lemma 23. If there exists a polynomial-time algorithm able to achieve advantage $2^{2} \delta$ in distinguishing between the distributions $\mathcal{D}_{1}(2)$ and $\mathcal{D}_{2}(2)$, then there exists a polynomial-time algorithm able to achieve advantage $\delta$ in the 2-Linear Problem.

Proof. If there exists a polynomial time algorithm $\mathcal{A}$ which distinguishes between $\mathcal{D}_{1}(2)$ and $\mathcal{D}_{2}(2)$ with advantage $2^{2} \delta$, we can construct a distinguisher for the 2 -Linear problem: $\mathcal{B}$. $\mathcal{B}$, upon receiving $g, g^{y_{1}}, g^{y_{2}}, g^{y_{1} c_{1}}, g^{y_{2} c_{2}}, g^{c_{1}+c_{2}+r}$, draws uniform random $\tilde{s}, b_{3}, \tilde{y}_{3}, \tilde{t}_{1}, \tilde{t}_{2}, \tilde{t}_{3}, \gamma_{1}, \gamma_{2} \leftarrow \mathbb{Z}_{q}$, then creates the set:

$$
\begin{aligned}
& g^{\tilde{s}}, \\
& g^{y_{1}}, \quad g^{y_{2}}, \quad g^{\tilde{y} 3}, \\
& g^{y_{1} c_{1}}, \quad g^{y_{2} c_{2}}, \quad\left(g^{c_{1}+c_{2}+r}\right)^{\tilde{y} 3}, \\
& \left(g^{y_{1} c_{1}}\right)^{-\frac{\tilde{s}}{\tilde{t}_{1}}}\left(g^{y_{1}}\right)^{\gamma_{1}}, \quad\left(g^{y_{2} r_{c}}\right)^{-\frac{\tilde{s}}{\tilde{t}_{2}}}\left(g^{y_{2}}\right)^{\gamma_{2}}, \quad g^{\tilde{y} b_{3}}, \\
& g^{\tilde{t}_{1}}, \quad g^{\tilde{t}_{2}}, \quad g^{\tilde{t}_{3}}, \\
& g^{\tilde{t}_{1} \gamma_{1}}, \quad g^{\tilde{t}_{2} \gamma_{2}}, \quad\left(g^{c_{1}+c_{2}+r}\right)^{\tilde{s}} g^{\tilde{t}_{3} b_{3}}
\end{aligned}
$$

then runs $\mathcal{A}$ on this input and returns the output of $\mathcal{A}$.

Notice that if $r=0$, this distribution is exactly $\mathcal{D}_{2}(2)$ (with $C=\left\{c_{1}, c_{2}\right\}, \tilde{y}_{1}=y_{1}, \tilde{y}_{2}=$ $y_{2}, b_{1}=-\frac{c_{1} s}{\tilde{t}_{1}}+\gamma_{1}$, and $\left.b_{2}=-\frac{c_{2} s}{\tilde{t}_{2}}+\gamma_{2}\right)$. If $r$ is instead random, this distribution is exactly $\mathcal{D}_{1}(2)$. Therefore, $\mathcal{B}$ will achieve the same advantage $2^{2} \delta$ as $\mathcal{A}$ (which is greater than $\delta$ ) in deciding the 2-Linear problem.

Lemma 24. For all integers $m \geq 2$, if there exists a polynomial-time algorithm able to achieve an advantage of $2^{m+1} \delta$ deciding between distributions $\mathcal{D}_{1}(m+1)$ and $\mathcal{D}_{2}(m+1)$, then either there exists a polynomial-time algorithm able to achieve an advantage of $2^{m} \delta$ in deciding between distributions $\mathcal{D}_{1}(m)$ and $\mathcal{D}_{2}(m)$ or there exists a polynomial time algorithm able to achieve an advantage of $\delta$ in the 2-Linear Problem. 
Proof. If there exists a polynomial time algorithm $\mathcal{A}$ which distinguishes between $\mathcal{D}_{1}(m+1)$ and $\mathcal{D}_{2}(m+1)$ with non-negligible advantage $2^{m+1} \delta$, we construct $\mathcal{B}$ : a distinguisher for $\mathcal{D}_{1}(m)$ and $\mathcal{D}_{2}(m)$.

$\mathcal{B}$, upon receiving

$$
\begin{aligned}
& g^{\tilde{s}}, \\
& g^{\tilde{y}_{1}}, \ldots, g^{\tilde{y}_{M-1}}, \\
& g^{\tilde{y}_{1} r_{1}}, \ldots, g^{\tilde{y}_{M-1} r_{M-1}}, \\
& g^{\tilde{y}_{1} b_{1}}, \ldots, g^{\tilde{y}_{M-1} b_{M-1}}, \\
& g^{\tilde{t}_{1}}, \ldots, g^{\tilde{t}_{M-1}}, \\
& g^{\tilde{s} r_{1}+\tilde{t}_{1} b_{1}}, \ldots, g^{\tilde{s} r_{M-1}+\tilde{t}_{M-1} b_{M-1}},
\end{aligned}
$$

where $M=2^{m}$, first draws $y_{1}^{*}, \ldots, y_{M-1}^{*}, \sigma_{1}, \ldots, \sigma_{M-1}, \gamma_{1}, \ldots, \gamma_{M-1}, \tilde{y}_{M}, \tilde{t}_{M}, b_{M}, c_{m+1} \leftarrow \mathbb{Z}_{q}$, and constructs the following set:

$g^{\tilde{s}}$

$g^{\tilde{y}_{1}}, \ldots, g^{\tilde{y}_{M-1}}, g^{\tilde{y}_{M}}$,

$\left(g^{\tilde{y}_{1}}\right)^{y_{1}^{*}}, \ldots,\left(g^{\tilde{y}_{M-1}}\right)^{y_{M-1}^{*}}$,

$g^{\tilde{y}_{1} r_{1}}, \ldots, g^{\tilde{y}_{M-1} r_{M-1}}, g^{\tilde{y}_{M} c_{m+1}}$,

$\left(g^{\tilde{y}_{1}}\right)^{y_{1}^{*} c_{m+1}}\left(g^{\tilde{y}_{1} r_{1}}\right)^{y_{1}^{*}}, \ldots,\left(g^{\tilde{y}_{M-1}}\right)^{y_{M-1}^{*} c_{m+1}}\left(g^{\tilde{y}_{M-1} r_{M-1}}\right)^{y_{M-1}^{*}}$,

$g^{\tilde{y}_{1} b_{1}}, \ldots, g^{\tilde{y}_{M-1} b_{M-1}}, g^{\tilde{y}_{M} b_{M}}$,

$\left(g^{\tilde{y}_{1} b_{1}}\right)^{y_{1}^{*}}\left(g^{\tilde{y}_{1}}\right)^{\sigma_{1} y_{1}^{*}}, \ldots,\left(g^{\tilde{y}_{M-1} b_{M-1}}\right)^{y_{M-1}^{*}}\left(g^{\tilde{y}_{M-1}}\right)^{\sigma_{M-1} y_{M-1}^{*}}$,

$g^{\tilde{t}_{1}}, \ldots, g^{\tilde{t}_{M-1}}, g^{\tilde{t}_{M}}$,

$g^{\tilde{t}_{1}}\left(g^{\tilde{y}_{1}}\right)^{\gamma_{1}}, \ldots, g^{\tilde{t}_{M-1}}\left(g^{\tilde{y}_{M-1}}\right)^{\gamma_{M-1}}$,

$g^{\tilde{s} r_{1}+\tilde{t}_{1} b_{1}}, \ldots, g^{\tilde{s} r_{M-1}+\tilde{t}_{M-1} b_{M-1}},\left(g^{\tilde{s}}\right)^{c_{m+1}} g^{\tilde{t}_{M} b_{M}}$,

$\left(g^{\tilde{s}}\right)^{c_{m+1}} g^{\tilde{s} r_{1}+\tilde{t}_{1} b_{1}}\left(g^{\tilde{t}_{1}}\right)^{\sigma_{1}}\left(g^{b_{1} \tilde{y}_{1}}\right)^{\gamma_{1}}\left(g^{\tilde{y}_{1}}\right)^{\sigma_{1} \gamma_{1}}, \ldots,\left(g^{\tilde{s}}\right)^{c_{m+1}} g^{\tilde{s} r_{M-1}+\tilde{t}_{M-1} b_{M-1}}\left(g^{\tilde{t}_{M-1}}\right)^{\sigma_{M-1}}\left(g^{b_{M-1} \tilde{y}_{M-1}}\right)^{\gamma_{M-1}}\left(g^{\tilde{y}_{M-1}}\right)^{\sigma_{M-1} \gamma_{M-1}}$

$=$

$g^{\tilde{s}}$,

$g^{\tilde{y}_{1}}, \ldots, g^{\tilde{y}_{M-1}}, g^{\tilde{y}_{M}}$,

$g^{\tilde{y}_{1} y_{1}^{*}}, \ldots, g^{\tilde{y}_{M-1} y_{M-1}^{*}}$,

$g^{\tilde{y}_{1} r_{1}}, \ldots, g^{\tilde{y}_{M-1} r_{M-1}}, g^{\tilde{y}_{M} c_{m+1}}$,

$g^{\tilde{y}_{1} y_{1}^{*}\left(r_{1}+c_{m+1}\right)}, \ldots, g^{\tilde{y}_{M-1} y_{M-1}^{*}\left(r_{M-1}+c_{m+1}\right)}$,

$g^{\tilde{y}_{1} b_{1}}, \ldots, g^{\tilde{y}_{M-1} b_{M-1}}, g^{\tilde{y}_{M} b_{M}}$,

$g^{\tilde{y}_{1} y_{1}^{*}\left(b_{1}+\sigma_{1}\right)}, \ldots, g^{\tilde{y}_{M-1} y_{M-1}^{*}\left(b_{M-1}+\sigma_{M-1}\right)}$,

$g^{\tilde{t}_{1}}, \ldots, g^{\tilde{t}_{M-1}}, g^{\tilde{t}_{M}}$,

$g^{\tilde{t}_{1}+\tilde{y}_{1} \gamma_{1}}, \ldots, g^{\tilde{t}_{M-1}+\tilde{y}_{M-1} \gamma_{M-1}}$,

$g^{\tilde{s} r_{1}+\tilde{t}_{1} b_{1}}, \ldots, g^{\tilde{s} r_{M-1}+\tilde{t}_{M-1} b_{M-1}}, g^{\tilde{s} c_{m+1}+\tilde{t}_{M} b_{M}}$,

$g^{\tilde{s}\left(r_{1}+c_{m+1}\right)+\left(\tilde{t}_{1}+\tilde{y}_{1} \gamma_{1}\right)\left(b_{1}+\sigma_{1}\right)}, \ldots, g^{\tilde{s}\left(r_{M-1}+c_{m+1}\right)+\left(\tilde{t}_{M-1}+\tilde{y}_{M-1} \gamma_{M-1}\right)\left(b_{M-1}+\sigma_{M-1}\right)}$ 
Notice that if $\mathcal{B}$ 's input is $\mathcal{D}_{2}(m)$, then the distribution of sets constructed by $\mathcal{B}$ is exactly $\mathcal{D}_{2}(m+1)$, where a new $c_{m+1}$ element is drawn and added to form the subsets of the new augmented set $C, \tilde{y}_{M+i}=\tilde{y}_{i} y_{i}^{*}, b_{M+i}=b_{i}+\sigma_{i}$, and $\tilde{t}_{M+i}=\tilde{t}_{i}+\tilde{y}_{i} \gamma_{i}$ for $i=1, \ldots, M-1$ which are all uniformly distributed at random. However, if $\mathcal{B}$ 's input is $\mathcal{D}_{1}(m)$, then the distribution of sets constructed by $\mathcal{B}$ is not exactly $\mathcal{D}_{1}(m+1)$.

Definition 25. Let $\mathcal{D}_{1}^{\prime}(m+1)$ be the distribution of sets created by $\mathcal{B}$ given input sets from $\mathcal{D}_{1}(m)$.

We have therefore only proved that if an algorithm is able to achieve advantage in distinguishing $\mathcal{D}_{1}^{\prime}(m+1)$ and $\mathcal{D}_{2}(m+1)$, then it can be used to achieve that same advantage in deciding between $\mathcal{D}_{1}(m)$ and $\mathcal{D}_{2}(m)$.

Fortunately, we can transition between $\mathcal{D}_{1}^{\prime}(m+1)$ and $\mathcal{D}_{1}(m+1)$ using a hybrid proof:

First we define $M=2^{m}$ hybrid distributions indexed by $(j)$ :

Definition 26. Let $\mathcal{D}_{1}^{\prime(j)}(m+1)$ be the distribution of:

$$
\begin{aligned}
& g^{\tilde{s}}, \\
& g^{\tilde{y}_{1}}, \ldots, g^{\tilde{y}_{M-1}}, g^{\tilde{y}_{M}}, \\
& g^{\tilde{y}_{M+1}}, \ldots, g^{\tilde{y}_{2 M-1}}, \\
& g^{\tilde{y}_{1} r_{1}}, \ldots, g^{\tilde{y}_{M-1} r_{M-1}}, g^{\tilde{y}_{M} c_{m+1}}, \\
& g^{\tilde{y}_{M+1}\left(r_{1}+c_{m+1}\right)}, \ldots, g^{\tilde{y}_{M+j}\left(r_{j}+c_{m+1}\right)}, g^{\tilde{y}_{M+j+1} r_{M+j+1}}, \ldots, g^{\tilde{y}_{2 M-1} r_{2 M-1}}, \\
& g^{\tilde{y}_{1} b_{1}}, \ldots, g^{\tilde{y}_{M-1} b_{M-1}}, g^{\tilde{y}_{M} b_{M}}, \\
& g^{\tilde{y}_{M+1} b_{M+1}}, \ldots, g^{\tilde{y}_{2 M-1} b_{2 M-1}}, \\
& g^{\tilde{t}_{1}}, \ldots, g^{\tilde{t}_{M-1}}, g^{\tilde{t}_{M}}, \\
& g^{\tilde{t}_{M+1}}, \ldots, g^{\tilde{t}_{2 M-1}}, \\
& g^{\tilde{s} r_{1}+\tilde{t}_{1} b_{1}}, \ldots, g^{\tilde{s} r_{M-1}+\tilde{t}_{M-1} b_{M-1}}, g^{\tilde{s} c_{m+1}+\tilde{t}_{M} b_{M}}, \\
& g^{\tilde{s}\left(r_{1}+c_{m+1}\right)+\tilde{t}_{M+1} b_{M+1}}, \ldots, g^{\tilde{s}\left(r_{j}+c_{m+1}\right)+\tilde{t}_{M+j} b_{M+j}}, g^{\tilde{s} r_{M+j+1}+\tilde{t}_{M+j+1} b_{M+j+1}}, \ldots, g^{\tilde{s} r_{2 M-1}+\tilde{t}_{2 M-1} b_{2 M-1}}
\end{aligned}
$$

for $j=0, \ldots, M-1$ where the $r_{M+j+i}$ are distributed uniformly at random in $\mathbb{Z}_{p}$ for $i=$ $1, \ldots M-j-1$

Notice that $\mathcal{D}_{1}^{\prime(0)}(m+1)=\mathcal{D}_{1}(m+1)$ and $\mathcal{D}_{1}^{\prime(M-1)}(m+1)=\mathcal{D}_{1}^{\prime}(m+1)$. So, if some adversary $\mathcal{A}$ could distinguish between $\mathcal{D}_{1}(m+1)$ and $\mathcal{D}_{1}^{\prime}(m+1)$ with non-negligible advantage $2^{m} \delta$, then:

$$
\left|\operatorname{Pr}\left[\mathcal{A}=1 \mid \mathcal{D}_{1}^{\prime(0)}(m+1)\right]-\operatorname{Pr}\left[\mathcal{A}=1 \mid \mathcal{D}_{1}^{\prime(M-1)}(m+1)\right]\right|=2^{m} \delta
$$

So, by the triangle inequality, there must exists some $j$ such that:

$$
\left|\operatorname{Pr}\left[\mathcal{A}=1 \mid \mathcal{D}_{1}^{\prime(j+1)}(m+1)\right]-\operatorname{Pr}\left[\mathcal{A}=1 \mid \mathcal{D}_{1}^{\prime(j)}(m+1)\right]\right| \geq \frac{2^{m} \delta}{M}=\delta
$$

Such an $\mathcal{A}$ can be used to construct a distinguisher for the 2-Linear Problem: $\mathcal{B}$ that achieves advantage $\delta$ :

$\mathcal{B}$, upon receiving $g, g^{y_{1}}, g^{y_{2}}, g^{y_{1} c_{1}}, g^{y_{2} c_{2}}, g^{c_{1}+c_{2}+r}$, relabels the elements as:

$$
g, g^{y_{1}}, g^{y_{2}}, g^{y_{1} r^{*}}, g^{y_{2} c_{m+1}}, g^{x}
$$


(defining $y_{1}=y_{1}, y_{2}=y_{2}, r^{*}=c_{1}, c_{m+1}=c_{2}$, and $\left.x=r^{*}+c_{m+1}+r\right) \mathcal{B}$ then draws

$$
\begin{aligned}
& \tilde{s}, \gamma_{j+1} \\
& \tilde{y}_{1}, \ldots, \tilde{y}_{j}, \tilde{y}_{j+2}, \ldots, \tilde{y}_{M-1} \\
& y_{M}^{*}, \ldots, y_{M+j}^{*}, \\
& \gamma_{M}, \ldots, \gamma_{M+j}, \\
& \tilde{y}_{M+j+1}, \ldots, \tilde{y}_{2 M-1}, \\
& \tilde{t}_{1}, \ldots, \tilde{t}_{2 M-1} \\
& b_{j+2}, \ldots, b_{2 M-1} \\
& r_{1}, \ldots, r_{j}, r_{j+2}, \ldots, r_{2 M-1}
\end{aligned}
$$

uniformly at random from $\mathbb{Z}_{q}$ and constructs:

$g^{\tilde{s}}$

$g^{\tilde{y}_{1}}, \ldots, g^{\tilde{y}_{j}}, g^{y_{1}}, g^{\tilde{y}_{j+2}}, \ldots, g^{\tilde{y}_{M-1}},\left(g^{y_{2}}\right)^{y_{M}^{*}}$,

$\left(g^{y_{2}}\right)^{y_{M+1}^{*}}, \ldots,\left(g^{y_{2}}\right)^{y_{M+j}^{*}}, g^{\tilde{y}_{M+j+1}}, \ldots, g^{\tilde{y}_{2 M-1}}$,

$g^{\tilde{y}_{1} r_{1}}, \ldots, g^{\tilde{y}_{j} r_{j}}, g^{y_{1} r^{*}}, g^{\tilde{y}_{j+2} r_{j+2}}, \ldots, g^{\tilde{y}_{M-1} r_{M-1}},\left(g^{y_{2} c_{m+1}}\right)^{y_{M}^{*}}$,

$\left(\left(g^{y_{2}}\right)^{y_{M+1}^{*}}\right)^{r_{1}}\left(g^{y_{2} c_{m+1}}\right)^{y_{M+1}^{*}}, \ldots,\left(\left(g^{y_{2}}\right)^{y_{M+j}^{*}}\right)^{r_{j}}\left(g^{y_{2} c_{m+1}}\right)^{y_{M+j}^{*}},\left(g^{x}\right)^{\tilde{y}_{M+j+1}}, g^{\tilde{y}_{M+j+2} r_{M+j+2}}, \ldots, g^{\tilde{y}_{2 M-1} r_{2 M-1}}$,

$g^{\tilde{y}_{1} b_{1}}, \ldots, g^{\tilde{y}_{j} b_{j}},\left(g^{y_{1} r^{*}}\right)^{-\frac{\tilde{s}}{t_{j+1}}}\left(g^{y_{1}}\right)^{\gamma_{j+1}}, g^{\tilde{y}_{j+2} b_{j+2}}, \ldots, g^{\tilde{y}_{M-1} b_{M-1}},\left(g^{y_{2}}\right)^{y_{M-1}^{*} b_{M-1}},\left(g^{y_{2}}\right)^{y_{M}^{*} \gamma_{M}}\left(g^{y_{2} c_{m+1}}\right)^{-y_{M}^{*} \frac{\tilde{s}}{t_{M}}}$

$\left(g^{y_{2}}\right)^{-y_{M+1}^{*}\left(\frac{\tilde{s} r_{1}}{t_{M+1}}-\gamma_{M+1}\right)}\left(g^{y_{2} c_{m+1}}\right)^{-y_{M+1}^{*} \frac{\tilde{s}}{t_{M+1}}}, \ldots,\left(g^{y_{2}}\right)^{-y_{M+j}^{*}\left(\frac{\tilde{s} r_{j}}{\hat{t}_{M+j}}-\gamma_{M+j}\right)}\left(g^{y_{2} c_{m+1}}\right)^{-y_{M+j}^{*} \frac{\tilde{s}}{\tilde{t}_{M+j}}}$,

$g^{\tilde{y}_{M+j+1} b_{M+j+1}}, \ldots, g^{\tilde{y}_{2 M-1} b_{2 M-1}}$,

$g^{\tilde{t}_{1}}, \ldots, g^{\tilde{t}_{M}}$,

$g^{\tilde{t}_{M+1}}, \ldots, g^{\tilde{t}_{2 M-1}}$,

$g^{\tilde{s} r_{1}+\tilde{t}_{1} b_{1}}, \ldots, g^{\tilde{s} r_{j}+\tilde{t}_{j} b_{j}}, g^{\tilde{t}_{j+1} \gamma_{j+1}}, g^{\tilde{s} r_{j+1}+\tilde{t}_{j+1} b_{j+1}}, \ldots, g^{\tilde{s} r_{M-1}+\tilde{t}_{M-1} b_{M-1}}, g^{\tilde{t}_{M} \gamma_{M}}$

$g^{\tilde{t}_{M+1} \gamma_{M+1}}, \ldots, g^{\tilde{t}_{M+j} \gamma_{M+j}},\left(g^{x}\right)^{\tilde{s}} g^{\tilde{t}_{M+j+1} b_{M+j+1}}, g^{\tilde{s} r_{M+j+2}+\tilde{t}_{M+j+2} b_{M+j+2}}, \ldots, g^{\tilde{s} r_{2 M-1}+\tilde{t}_{2 M-1} b_{2 M-1}}$

where $\tilde{y}_{j+1}=y_{1}, r_{j+1}=r^{*}, b_{j+1}=-\frac{\tilde{s} r^{*}}{\tilde{t}_{j+1}}+\gamma_{j+1}, b_{M}=-\frac{\tilde{s} c_{m+1}}{\tilde{t}_{M}}+\gamma_{M}$,

and the $b_{M+i}=-\frac{\tilde{s}\left(r_{i}+c_{m+1}\right)}{\tilde{t}_{M+i}}+\gamma_{M+i}$ for $i=1, \ldots, j$ and $\tilde{y}_{M+i}=y_{2} \tilde{y}_{M+i}^{*}$ for $i=0, \ldots, j$ are all distributed uniformly at random in $\mathbb{Z}_{p}$.

$\mathcal{B}$ then runs $\mathcal{A}$ on this input and outputs the same.

Note that if $x=r^{*}+c_{m+1}+0$, then $\mathcal{B}$ has sampled an instance of $\mathcal{D}_{1}^{(j+1)}(m+1)$. Otherwise, if $x=r^{*}+c_{m+1}+r$ for a uniform random $r$ it has sampled an instance of $\mathcal{D}_{1}^{(j)}(m+1)$. So, $\mathcal{B}$ will enjoy the same advantage $\delta$ of $\mathcal{A}$ but in deciding the 2-Linear Problem.

We assumed there is a polynomial time algorithm $\mathcal{A}$ which distinguishes between $\mathcal{D}_{1}(m+1)$ and $\mathcal{D}_{2}(m+1)$ with advantage $2^{m+1} \delta$. By the triangle inequality, then $\mathcal{A}$ must be able to be used to either achieve advantage $2^{m} \delta$ in distinguishing between instances of $\mathcal{D}_{1}(m+1)$ and $\mathcal{D}_{1}^{\prime}(m+1)$ or achieve advantage $2^{m} \delta$ in distinguishing between instances of between $\mathcal{D}_{1}^{\prime}(m+1)$ and $\mathcal{D}_{2}(m+1)$.

In the first case, if $\mathcal{A}$ can be used to achieve advantage $2^{m} \delta$ in distinguishing between instances of $\mathcal{D}_{1}(m+1)$ and $\mathcal{D}_{1}^{\prime}(m+1)$, then we showed in the first proof how such an algorithm could be used to distinguish between $\mathcal{D}_{1}(m)$ and $\mathcal{D}_{2}(m)$ with the same advantage $\left(2^{m} \delta\right)$. 
In the second case, if $\mathcal{A}$ can be used to achieve advantage $2^{m} \delta$ in distinguishing between instances of $\mathcal{D}_{1}^{\prime}(m+1)$ and $\mathcal{D}_{2}(m+1)$, then we showed in the second proof how such an algorithm could be used to break the 2-Linear problem with advantage $\frac{2^{m} \delta}{M}=\delta$.

Therefore, if there is a polynomial time algorithm $\mathcal{A}$ which distinguishes between $\mathcal{D}_{1}(m+1)$ and $\mathcal{D}_{2}(m+1)$ with advantage $2^{m+1} \delta$, then either there exists a polynomial-time algorithm able to achieve an advantage of $2^{m} \delta$ in deciding between distributions $\mathcal{D}_{1}(m)$ and $\mathcal{D}_{2}(m)$ or there exists a polynomial time algorithm able to achieve an advantage of $\delta$ in the 2-Linear Problem.

Now we have all the ingredients necessary for the main statement:

Lemma 27. The distributions $\mathcal{D}_{1}(k)$ and $\mathcal{D}_{2}(k)$ are computationally indistinguishable under the 2-Linear computational hardness assumption if $k=O(\lg$ poly $(\lambda))$.

Proof. We have shown that for all integers $m \geq 2$, if there exists a polynomial-time algorithm able to achieve an advantage of $2^{m+1} \delta$ deciding between distributions $\mathcal{D}_{1}(m+1)$ and $\mathcal{D}_{2}(m+1)$, then either there exists a polynomial-time algorithm able to achieve an advantage of $2^{m} \delta$ in deciding between distributions $\mathcal{D}_{1}(m)$ and $\mathcal{D}_{2}(m)$ or there exists a polynomial time algorithm able to achieve an advantage of $\delta$ in the 2-Linear Problem. We have also shown that if there exists a polynomial-time algorithm able to achieve advantage $2^{2} \delta$ in distinguishing between the distributions $\mathcal{D}_{1}(2)$ and $\mathcal{D}_{2}(2)$, then there exists a polynomial-time algorithm able to achieve advantage $\delta$ in the 2-Linear Problem. By induction, it follows that for all $m$, if an algorithm is able to achieve an advantage of $2^{m} \delta$ in distinguishing between distributions $\mathcal{D}_{1}(m)$ and $\mathcal{D}_{2}(m)$, then that algorithm can be used to achieve advantage $\delta$ in the 2-Linear problem. Equivalently, if an algorithm is able to achieve an advantage of $\delta^{\prime}$ in distinguishing between distributions $\mathcal{D}_{1}(m)$ and $\mathcal{D}_{2}(m)$, then that algorithm can be used to achieve advantage $\frac{\delta^{\prime}}{2^{m}}$ in the 2-Linear problem.

If $k=O(\lg \operatorname{poly}(\lambda))$, then any algorithm $\mathcal{A}$ able to achieve non-negligible advantage $\delta$ in distinguishing between $\mathcal{D}_{1}(k)$ and $\mathcal{D}_{2}(k)$ can be used to achieve non-negligible advantage $\Omega\left(\frac{\delta}{\operatorname{poly}(\lambda)}\right)$ in the 2-Linear problem. This violates our 2-Linear Assumption, so no such algorithm $\mathcal{A}$ can exist.

\section{Concluding Remarks}

We have presented a composite order KP-ABE scheme proven fully secure under the DLIN assumption and additional subgroup decision type assumptions. The scheme allows a bound of $2^{k}-1$ attribute-uses in an access policy, where the number of group elements required in the public parameters per attribute-use grows polynomially with $k$. An interesting question for future work is whether the ciphertext sizes can be significantly reduced (our scheme has ciphertexts still growing linearly in size with $2^{k}-1$ ). We have chosen to demonstrate our techniques on a KP-ABE scheme, though we note that they are equally applicable to the CPABE setting. The core of CP-ABE schemes often mirror the structure of KP-ABE schemes, and would benefit similarly from the reduced public parameter size our lemma enables. Finally, our bilinear entropy expansion lemma is not restricted to the ABE setting, and we suspect it may have applications to other cryptographic primitives. Primitive structure can be built around the lemma's core components of $\left\{g^{t_{K}}, g^{t_{K} A_{K}}\right\}$, which can be plugged in to replace a need for independent random group elements. Our composite order KP-ABE scheme demonstrates this usage. 


\section{References}

[1] N. Attrapadung. Dual system encryption via doubly selective security: Framework, fully secure functional encryption for regular languages, and more. In EUROCRYPT, pages $557-577,2014$.

[2] M. Raykova B. Parno and V. Vaikuntanathan. How to delegate and verify in public: Verifiable computation from attribute-based encryption. In TCC, pages 422-439, 2012.

[3] A. Beimel. Secure schemes for secret sharing and key distribution. PhD thesis, Israel Institute of Technology, Technion, Haifa, Israel, 1996.

[4] John Bethencourt, Amit Sahai, and Brent Waters. Ciphertext-policy attribute-based encryption. In Proceedings of the IEEE Symposium on Security and Privacy, pages 321-334.

[5] D. Boneh and M. Franklin. Identity based encryption from the weil pairing. In CRYPTO, pages 213-229, 2001.

[6] D. Boneh, E. Goh, and K. Nissim. Evaluating 2-dnf formulas on ciphertexts. In TCC, pages $325-342,2005$.

[7] M. Chase. Multi-authority attribute based encryption. In Theory of Cryptography, 4th Theory of Cryptography Conference, TCC 2007, Amsterdam, The Netherlands, February 21-24, 200\%, Proceedings, pages 515-534, 2007.

[8] M. Chase and S. S. M. Chow. Improving privacy and security in multi-authority attributebased encryption. In Proceedings of the 2009 ACM Conference on Computer and Communications Security, pages 121-130, 2009.

[9] M. Chase and S. Meiklejohn. Déjà Q: using dual systems to revisit q-type assumptions. In EUROCRYPT, pages 622-639, 2014.

[10] J. Chen and H. Wee. Fully, (almost) tightly secure IBE and dual system groups. In CRYPTO, pages 435-460, 2013.

[11] C. Cocks. An identity based encryption scheme based on quadratic residues. In Proceedings of the 8th IMA International Conference on Cryptography and Coding, pages 26-28, 2001.

[12] Y. Dodis, A. B. Lewko, B. Waters, and D. Wichs. Storing secrets on continually leaky devices. In FOCS, pages 688-697, 2011.

[13] S. Garg, C. Gentry, S. Halevi, A. Sahai, and B. Waters. Attribute-based encryption for circuits from multilinear maps. In CRYPTO, pages 479-499, 2013.

[14] S. Garg, C. Gentry, S. Halevi, and M. Zhandry. Fully secure attribute based encryption from multilinear maps. IACR Cryptology ePrint Archive, 2014:622, 2014.

[15] S. Goldwasser, Y. T. Kalai, R. A. Popa, V. Vaikuntanathan, and N. Zeldovich. How to run turing machines on encrypted data. In Advances in Cryptology - CRYPTO 2013 - 33rd Annual Cryptology Conference, Santa Barbara, CA, USA, August 18-22, 2013. Proceedings, Part II, pages 536-553, 2013.

[16] S. Gorbunov, V. Vaikuntanathan, and H. Wee. Attribute-based encryption for circuits. In STOC, pages 545-554, 2013. 
[17] V. Goyal, A. Jain, O. Pandey, and A. Sahai. Bounded ciphertext policy attribute-based encryption. In ICALP, 2008.

[18] V. Goyal, O. Pandey, A. Sahai, and B. Waters. Attribute based encryption for fine-grained access control of encrypted data. In ACM conference on Computer and Communications Security, pages 89-98, 2006.

[19] A. Lewko, T. Okamoto, A. Sahai, K. Takashima, and B. Waters. Fully secure functional encryption: Attribute-based encryption and (hierarchical) inner product encryption. In EUROCRYPT, pages 62-91, 2010.

[20] A. Lewko, Y. Rouselakis, and B. Waters. Achieving leakage resilience through dual system encryption. In $T C C$, pages 70-88, 2011.

[21] A. Lewko and B. Waters. New techniques for dual system encryption and fully secure hibe with short ciphertexts. In TCC, pages 455-479, 2010.

[22] A. Lewko and B. Waters. Decentralizing attribute-based encryption. In EUROCRYPT, pages 568-588, 2011.

[23] A. Lewko and B. Waters. Unbounded hibe and attribute-based encryption. In EUROCRYPT, pages 547-567, 2011.

[24] A. B. Lewko, M. Lewko, and B. Waters. How to leak on key updates. In STOC, pages 725-734, 2011.

[25] A. B. Lewko and B. Waters. Efficient pseudorandom functions from the decisional linear assumption and weaker variants. In Proceedings of the 2009 ACM Conference on Computer and Communications Security, pages 112-120, 2009.

[26] A. B. Lewko and B. Waters. New proof methods for attribute-based encryption: Achieving full security through selective techniques. In CRYPTO, pages 180-198, 2012.

[27] M. Naor and O. Reingold. Number-theoretic constructions of efficient pseudo-random functions. In FOCS, pages 458-467, 1997.

[28] T. Okamoto and K. Takashima. Fully secure functional encryption with general relations from the decisional linear assumption. In CRYPTO, pages 191-208, 2010.

[29] T. Okamoto and K. Takashima. Fully secure unbounded inner-product and attribute-based encryption. In ASIACRYPT, pages 349-366, 2012.

[30] T. Okamoto and K. Takashima. Decentralized attribute-based signatures. In $P K C$, pages 125-142, 2013.

[31] R. Ostrovksy, A. Sahai, and B. Waters. Attribute based encryption with non-monotonic access structures. In ACM conference on Computer and Communications Security, pages 195-203, 2007.

[32] A. Sahai and B. Waters. Fuzzy identity based encryption. In EUROCRYPT, pages 457-473, 2005.

[33] B. Waters. Dual system encryption: realizing fully secure ibe and hibe under simple assumptions. In CRYPTO, pages 619-636, 2009. 
[34] B. Waters. Ciphertext-policy attribute-based encryption: An expressive, efficient, and provably secure realization. In $P K C$, pages 53-70, 2011.

[35] H. Wee. Dual system encryption via predicate encodings. In TCC, pages 616-637, 2014. 